EPJ Web of Conferences 22, 00014 (2012)

DOI: $10.1051 /$ epjconf/20122200014

(C) Owned by the authors, published by EDP Sciences, 2012

\title{
Applications of symmetries in superconductivity
}

\author{
M. Houzet \\ SPSMS, UMR-E 9001, CEA-INAC/UJF-Grenoble 1, 38054 Grenoble, France
}

\section{INTRODUCTION}

Superconductivity was discovered by Kamerlingh Onnes in 1911. It is characterized by two major properties: i) the electrical resistance exactly vanishes and ii) the magnetic field lines are expelled outside the superconducting body (Meissner effect). Both properties are potentially useful for many applications, in particular for storage and dissipationless transport of electrical energy. However, though superconductivity has been observed in many metals, it has been limited to low temperatures so far. Moreover, it cannot sustain too large external magnetic fields or current densities. Applications have thus been limited to quite specific, though significant areas (superconducting electromagnets used for Magnetic Resonance Imaging, for instance).

On the fundamental research side, superconductivity has fascinated many physicists, in particular because it is a direct manifestation of quantum coherence on the macroscopic scale. The theories that have been invented to explain this phenomenon have then irrigated other areas including condensed matter, nuclear, and particle physics. The aim of these lecture notes is to highlight that the concept of symmetry has been essential in making these advances. Section 2 will present the phenomenological description of superconductivity. It will allow ascribing it to a spontaneous gauge symmetry breaking in the frame of the Landau theory of phase transitions. In Section 3, the microscopic theory of superconductivity will be introduced. Unconventional superconductors where an additional symmetry breaking takes place at the phase transition will be contrasted with conventional superconductors where only the gauge symmetry breaking takes place. Physical signatures that allow distinguishing conventional and unconventional superconductors will be discussed. Section 4 will conclude by presenting active fields of research in superconductivity where the concepts of symmetry are still being used.

For the clarity of the presentation, strong simplifications will be made in the analysis and many aspects of the theory will be put aside. The reader may consult the textbooks [1-3] for further reading and references. A detailed presentation of the aspects related with symmetries can be found in Refs. $[4,5]$.

\section{PHENOMENOLOGICAL THEORY - SYMMETRY BREAKING IN SUPERCONDUCTORS}

In this section, we show that the concept of spontaneous gauge symmetry breaking allows describing phenomenologically major properties of superconductivity. In particular, a complex order parameter is introduced and the measurability of its phase is discussed in the frame of the Josephson effect.

\subsection{London theory - perfect diamagnetic screening}

A step toward the understanding of both the zero electrical resistance and magnetic field expulsion in superconductors was made by London and London in 1935. It was assumed that the free energy in the

This is an Open Access article distributed under the terms of the Creative Commons Attribution-Noncommercial License 3.0, which permits unrestricted use, distribution, and reproduction in any noncommercial medium, provided the original work is properly cited. 


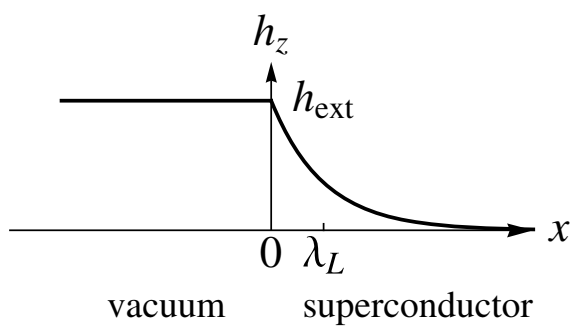

Figure 1. Magnetic field penetration in a superconductor.

superconducting state is

$$
F=F_{s}+\int d \boldsymbol{x}\left(\frac{\boldsymbol{h}^{2}}{8 \pi}+\frac{1}{2} m n_{s} \boldsymbol{v}^{2}\right) .
$$

Here, the first term is the energy of the superconducting state in the absence of magnetic field, the second term is the magnetic energy associated with an internal magnetic field $\boldsymbol{h}(\boldsymbol{x})$ at position $\boldsymbol{x}$, and the last term is the kinetic energy associated with a "superfluid" density $n_{s}$ of electrons with mass $m$ and velocity $\boldsymbol{v}(\boldsymbol{x})$, that may also vary spatially. The bold assumption consists in assuming that $n_{s}$ is a significant fraction of the conduction electrons and, thus, that the kinetic energy term plays a macroscopic role. This is in contrast with the conventional diamagnetic effect in normal metals which is very small. Note that the term "superfluid" is coined by analogy with the phenomenon of superfluidity in ${ }^{4} \mathrm{He}$ (bosonic atoms composed of an even number of 2 protons and 2 neutrons) which is understood to originate from a Bose-Einstein condensation of all atoms in a single quantum state. Nevertheless, electrons are fermions and the connection between both phenomena was not clear before the advent of a microscopic theory of superconductivity.

Now, the Maxwell equations for the magnetic field read

$$
\begin{aligned}
\operatorname{div} \boldsymbol{h} & =0, \\
\operatorname{rot} \boldsymbol{h} & =(4 \pi / c) \boldsymbol{j}_{s},
\end{aligned}
$$

where $\boldsymbol{j}_{s}=e n_{s} \boldsymbol{v}$ is the charge current density ( $e$ is the electron charge). With Eq. (2.2b), the free energy (2.1) reads

$$
F=F_{s}+\frac{1}{8 \pi} \int d \boldsymbol{x}\left[\boldsymbol{h}^{2}+\lambda_{L}^{2}(\operatorname{rot} \boldsymbol{h})^{2}\right]
$$

where $\lambda_{L}=\left(m c^{2} / 4 \pi n_{s} e^{2}\right)^{1 / 2}$. Minimizing Eq. (2.3) with respect to $\boldsymbol{h}$, one obtains the equation

$$
\boldsymbol{h}+\lambda_{L}^{2} \operatorname{rot} \operatorname{rot} \boldsymbol{h}=0 .
$$

The London theory then allows explaining superconductivity. Indeed, according to Eq. (2.4) together with (2.2a), an external magnetic field $\boldsymbol{h}_{\text {ext }}$ applied normally to the surface of a superconductor decays exponentially inside it: $\boldsymbol{h}(x)=\boldsymbol{h}_{\mathrm{ext}} e^{-x / \lambda_{L}}$, where $x$ is a coordinate normal to the interface so that the superconductor occupies the half-space at $x>0$, see Fig. 1. Thus, the magnetic field is perfectly screened in the superconductor over a characteristic scale $\lambda_{L}$, the magnetic penetration depth. This result explains the Meissner effect by the circulation of a dissipationless current parallel to the interface with vacuum that opposes to the field penetration: this is the perfect diamagnetic screening.

It is instructive to write the London equation in a specific gauge. Introducing the vector potential $\boldsymbol{A}$ such that $\boldsymbol{h}=\operatorname{rot} \boldsymbol{A}$ and using Eq. (2.2b) allows writing Eq. (2.4) as $\operatorname{rot}\left[\boldsymbol{A}+\left(4 \pi \lambda_{L}^{2} / c\right) \boldsymbol{j}_{s}\right]=0$. Then, as the current conservation imposes $\operatorname{div} \boldsymbol{j}_{s}=0$, the relation $\operatorname{div}\left[\boldsymbol{A}+\left(4 \pi \lambda_{L}^{2} / c\right) \boldsymbol{j}_{s}\right]=0$ also holds in a 
gauge in which $\operatorname{div} \boldsymbol{A}=0$. Thus, we find:

$$
\boldsymbol{j}_{s}=-\frac{n_{s} e^{2}}{m c} \boldsymbol{A}
$$

in this gauge. This equation is very different from the Ohm's law in metals that reads:

$$
\boldsymbol{j}_{n}=\sigma \boldsymbol{E}=-\frac{\sigma}{c} \frac{\partial \boldsymbol{A}}{\partial t}
$$

where $\sigma$ is the conductivity in normal state.

\subsection{Ginzburg-Landau theory}

The transition from normal to superconducting state is of the second order. For instance the specific heat has a characteristic jump when the temperature goes across the superconducting critical temperature. This observation led Ginzburg and Landau to generalize the Landau theory for second order phase transitions (see chapter by Toledano) to superconductivity in 1950.

For this, they introduced the Ginzburg-Landau (GL) free energy functional

$$
\left.F[\eta]=F_{n}+\left.\int d \boldsymbol{x}\{\alpha \mid \eta(\boldsymbol{x}))\right|^{2}+\frac{\beta}{2}|\eta(\boldsymbol{x})|^{4}+K\left|\left[-i \nabla-\frac{2 e}{\hbar c} \boldsymbol{A}(\boldsymbol{x})\right] \eta(\boldsymbol{x})\right|^{2}+\frac{\boldsymbol{h}(\boldsymbol{x})^{2}}{8 \pi}\right\},
$$

where $F_{n}$ is the normal-state free energy in absence of magnetic field. $F[\eta]$ depends on the complex order parameter $\eta(\boldsymbol{x})=|\eta(\boldsymbol{x})| e^{i \theta(\boldsymbol{x})}$. The modulus $|\eta|$ measures the density of superfluid electrons $n_{s} \propto$ $|\eta|^{2}$; the phase $\theta$ accounts for the quantum coherence of the superconducting state. $\eta$ was introduced by similarity with the complex wavefunction in the Schrödinger equation.

The form of Equation (2.7) is constrained by symmetry arguments: It is chosen so that it remains invariant under an arbitrary, constant phase multiplication: $\eta(\boldsymbol{x}) \rightarrow e^{i \theta_{0}} \eta(\boldsymbol{x})$. This is the global $U(1)$ gauge symmetry. ${ }^{1}$ Moreover, the spatial symmetries in cubic crystals determine the isotropic form of the gradient term in Eq. (2.7), while $K$ would be a tensor in crystals with a different symmetry. The transition to the superconducting phase is signaled by the change of sign of the coefficient $\alpha=\alpha_{0}\left(T-T_{c}\right) / T_{c}$ at temperatures $T$ below the superconducting critical temperature $T_{c}$. Note also that the coefficient $\beta$ in front of the quartic term $\propto|\eta|^{4}$ is positive for the free energy to well behave at large values of $|\eta|$. Also, $K>0$ expresses that a homogenous state with $\eta=$ const is favored at zero magnetic field.

Note that a free energy functional that depends on a complex order parameter is common to all superfluid transitions; for instance, an equation similar to (2.7) is known as the Gross-Pitaevskii equation in the context of the Bose-Einstein condensation.

A specific feature to superconductivity is that Equation (2.7) contains the effect of the magnetic field both through the magnetic energy term, $\boldsymbol{h}^{2} / 8 \pi$, and the covariant derivative, $\nabla-(2 i e / \hbar c) \boldsymbol{A}$, in the gradient term. The later is again introduced by analogy with the Schrödinger equation for charged particles in a magnetic field. The charge $2 e$ that appears there already anticipates the role of electron pairs in the microscopic explanation of superconductivity (see Sec. 3). It cannot be deduced from a phenomenological theory. Then, in addition to the global $U(1)$ gauge symmetry, Eq. (2.7) has also a local (electromagnetic) gauge symmetry: it remains invariant under the simultaneous electromagnetic gauge transformation that leaves the Maxwell equations for $\boldsymbol{h}$ invariant:

$$
\boldsymbol{A} \rightarrow \boldsymbol{A}+\nabla \chi
$$

where $\chi(\boldsymbol{x})$ is an arbitrary scalar field, and

$$
\eta \rightarrow \eta \exp (2 i e \chi / \hbar c) .
$$

\footnotetext{
${ }^{1} U(1)$ is the group of complex numbers $e^{i \theta_{0}}\left(\theta_{0}\right.$ real), with unity modulus.
} 


\section{EPJ Web of Conferences}

The consequences of the coupling between the superconducting order parameter and the local magnetic field will be examined in Sec. 2.3.

Finally, Equation (2.7) is invariant under time reversal symmetry, with $\boldsymbol{A} \rightarrow-\boldsymbol{A}$ and $\eta \rightarrow \eta^{*}$. $^{2}$

Equation (2.7) is a lowest order expansion of the free energy up to quadratic terms in the gradient expansion and the magnetic field, and quartic terms in $|\eta|$. Such an expansion has been justified soon after the microscopic theory of superconductivity - it is valid at temperatures close to $T_{c}$ and at small magnetic fields. that is,

The order parameter that extremizes the free energy (2.7) is determined by the equation $\delta F / \delta \eta^{*}=0$,

$$
\alpha \eta+\beta|\eta|^{2} \eta-K\left(\nabla-\frac{2 i e}{\hbar c} \boldsymbol{A}\right)^{2} \eta=0 .
$$

Equation (2.10) is similar to the Schrödinger equation for a particle with charge $2 e$, and it also includes a nonlinear (cubic) term. In the absence of magnetic field, the solution at $T>T_{c}(\alpha>0)$ is $\eta_{0}=0$. Thus, it trivially remains invariant under all symmetries in the system. At $T<T_{c}(\alpha<0)$, this solution corresponds to a maximum of the free energy and it is unstable. Instead, the stable solution is $\eta_{0}=\left|\eta_{0}\right| e^{i \theta}$, with $\left|\eta_{0}\right|^{2}=-\alpha / \beta$ and a given phase $\theta$. Thus it is not invariant under constant phase multiplication: the global $U(1)$ gauge symmetry is broken in the superconducting state.

We can now calculate the energy per unit volume gained in superconducting state (the condensation energy). At $T>T_{c}$ and $\eta=0$, Eq. (2.7) reduces to $F_{n}$. At $T<T_{c}$, the solution for $\eta$ is inserted in Eq. (2.7) and one gets $F_{s}=F_{n}-\alpha^{2} / 2 \beta$. This results in a jump in the specific heat $C=-T\left(\partial^{2} F / \partial T^{2}\right)$ at $T_{c}: \Delta C \equiv C_{s}-C_{n}=\alpha_{0}^{2} / \beta T_{c}$ as expected for a second order phase transition.

Another consequence of Eq. (2.10) is that spatial inhomogeneities in the order parameter are suppressed on a length $\xi(T)=\sqrt{K /|\alpha|}$, which is called the superconducting coherence length. Note that $\xi(T) \propto\left|T_{c}-T\right|^{-1 / 2}$ diverges at the transition.

Let us mention that generalized GL functionals where the coefficients $K$ and $\beta$ have negative signs, and higher order terms $\propto|\eta|^{6},\left|\nabla^{2} \eta\right|^{2}$, and $|\eta|^{2}|\nabla \eta|^{2}$ are added to Eq. (2.7), have also been proposed [6]. In the first case, the transition from normal to superconducting state changes from the second to the first order; the second case signals an instability toward a spontaneously modulated superconducting state, known as the Fulde-Ferrell-Larkin-Ovchinnikov state.

\subsection{Response to an electromagnetic field}

The GL free energy also describes the coupling between the superconducting order parameter and the magnetic field. The Maxwell equation is obtained from the condition $\delta F / \delta \boldsymbol{A}=0$, which yields:

$$
-\frac{2 e K}{\hbar c}\left[\eta^{*}\left(-i \nabla-\frac{2 e}{\hbar c} \boldsymbol{A}\right) \eta+\text { c.c. }\right]+\frac{1}{4 \pi} \operatorname{rot} \operatorname{rot} \boldsymbol{A}=0 \text {. }
$$

Equation (2.11) can be presented equivalently in the form (2.2b), with the superfluid current density

$$
\dot{j}_{s}=-\frac{2 i e K}{\hbar}\left(\eta^{*} \nabla \eta-\eta \nabla \eta^{*}\right)-\frac{8 e^{2} K}{\hbar^{2} c}|\eta|^{2} \boldsymbol{A} .
$$

Introducing $\eta=|\eta| e^{i \theta}$, we express Eq. (2.12) as:

$$
\boldsymbol{j}_{s}=\frac{4 e K}{\hbar}|\eta|^{2}\left(\nabla \theta-\frac{2 e}{\hbar c} \boldsymbol{A}\right) \text {. }
$$

\footnotetext{
2 The latter transformation can also be inferred by analogy with the time-reversed wavefunction $\psi(-t)=\psi(t)^{*}$ in the Schrödinger equation $i \hbar \partial_{t} \psi(t)=\mathcal{H} \psi(t)$ with Hamiltonian $\mathcal{H}$.
} 


\section{Contribution of Symmetries in Condensed Matter}

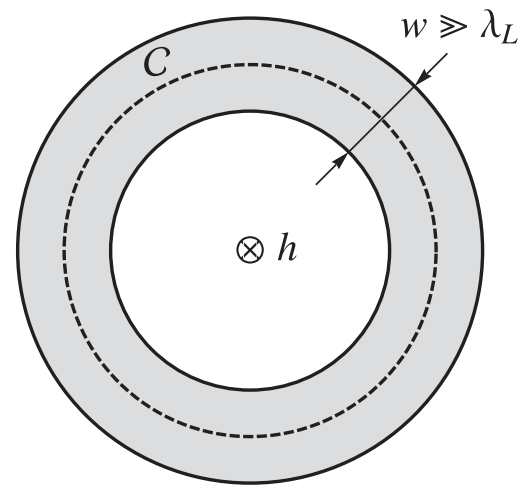

Figure 2. Superconducting ring geometry.

Solving Eq. (2.10) at small magnetic field is done most conveniently in the gauge with div $\boldsymbol{A}=0$ by separating $\eta$ into its modulus and phase. It is straightforward to show that corrections to the solution $\eta_{0}$ only appear at second order in $\boldsymbol{A}$. Thus Eq. (2.13) simplifies into the London equation (2.5) in this regime. Thereby it demonstrates that the Meissner effect and zero resistance state are also described by the GL theory. Moreover, the comparison of both equations allows relating the penetration depth to the parameters of the GL functional: $\lambda_{L}=\left(\hbar^{2} c^{2} \beta / 32 \pi e^{2}|\alpha| K\right)^{1 / 2}$. Thus, $\lambda_{L}(T) \propto\left(T_{c}-T\right)^{-1 / 2}$ like the coherence length. In particular, the ratio

$$
\kappa \equiv \frac{\lambda}{\xi}=\frac{\hbar c}{2|e| K} \sqrt{\frac{\beta}{8 \pi}}
$$

is temperature independent. It is called the GL parameter and it compares the characteristic scales for spatial variations of the magnetic field and order parameter, respectively.

The GL theory not only allows retrieving the London theory and its physical implications, but it can also predict new phenomena. For instance, let us consider a superconducting ring with a width $w$ larger than $\lambda_{L}$ (see Fig. 2) so that the magnetic field is completely expelled from the region deep inside, cf. Sec. 2.1. Thus, there is a contour $\mathcal{C}$ along the ring where the current (2.13) vanishes, and

$$
0=\oint_{\mathcal{C}} d \boldsymbol{\ell} \cdot \boldsymbol{j}_{s}=\frac{4 e K}{\hbar}\left|\eta_{0}\right|^{2}\left(\Delta \theta-\frac{2 e}{\hbar c} \Phi\right)
$$

Here, $\Delta \theta$ is the amount by which the phase changes when position varies along the ring back to the initial point; $\Phi=\oint_{\mathcal{C}} d \boldsymbol{l} . \boldsymbol{A}=\iint d \boldsymbol{S} . \boldsymbol{h}$ is the magnetic flux piercing the ring. As the order parameter should be single-valued, the relation $\Delta \theta=2 n \pi$, with integer $n$, must hold. Thus the ring encloses an integer number of superconducting flux quanta,

$$
\Phi=n \Phi_{0},
$$

where $\Phi_{0}=h c /(2|e|)$ is a combination of fundamental constants. Simultaneously, an equilibrium persistent current flows along the ring.

Another achievement of the GL theory is that it explains the existence of two kinds of superconductors. In type-I superconductors with $\kappa<1 / \sqrt{2}$, the transition from normal to superconducting state at finite magnetic field is of the first order. It occurs at a critical field $H_{c}$ when the condensation energy in Meissner state, $-\alpha^{2} / 2 \beta$, and the magnetic energy in normal state, $-H_{c}^{2} / 8 \pi{ }^{3}$

\footnotetext{
${ }^{3}$ Finding thermodynamic potentials in magnetic substances is a subtle issue. Indeed, the free energy (2.7) should be used at fixed temperature and internal field $\boldsymbol{h}$. However, the latter condition is impracticable. In experiments, the magnetic field is rather created by the circulation of a current in external coils surrounding the sample, which controls the external magnetic field $\boldsymbol{h}_{\text {ext }}$. For
} 
equal each other. That is,

$$
H_{c}=\left(\frac{4 \pi \alpha_{0}^{2}}{\beta T_{c}^{2}}\right)^{1 / 2}\left(T_{c}-T\right) .
$$

On the other hand, type-II superconductors with $\kappa>1 / \sqrt{2}$ are characterized by two superconducting states: a Meissner state at low field $H<H_{c 1}\left(H_{c 1}\right.$ is the lower critical field) and a mixed state at intermediate fields $H_{c 1}<H<H_{c 2}$ ( $H_{c 2}$ is the upper critical field) where the magnetic field penetrates partially inside the superconductor in the form of vortex lines. The normal-to-superconducting phase transition at $H_{c 2}$ is of the second order. To determine $H_{c 2}(T)$, we look for a solution of Eq. (2.10) with $\eta \rightarrow 0$, thus neglecting the cubic term. As the magnetic field $\boldsymbol{h}=H_{c 2} \hat{z}$ can be described with a vector potential $\boldsymbol{A}=H_{c 2} x \hat{\boldsymbol{y}}$, an order parameter that depends on $x$ only can be sought, with Eq. (2.10) reading

$$
-K \frac{\partial^{2} \eta}{\partial x^{2}}+K\left(\frac{2 e H_{c 2}}{\hbar c}\right)^{2} x^{2} \eta=-\alpha \eta .
$$

Equation (2.18) looks like the Schrödinger equation for an harmonic oscillator. ${ }^{4}$ With this analogy, we deduce that the highest field at which superconductivity exists is

$$
H_{c 2}=\frac{\hbar c}{2|e|} \frac{\alpha_{0}}{K T_{c}}\left(T_{c}-T\right)
$$

(it corresponds to $n=0$ in footnote 4 ). That is,

$$
H_{c 2}=H_{c} \kappa \sqrt{2}
$$

which is indeed larger than $H_{c}$ in type-II superconductors. ${ }^{5}$

In the mixed state, each vortex line has a normal vortex core with radius $\sim \xi$. As the upper critical field also reads: $H_{c 2}=\Phi_{0} /\left(2 \pi \xi^{2}\right)$, it means that superconductivity is destroyed when the cores overlap each other. The cores are surrounded by a supercurrent so that the vortices carry precisely one flux quantum, for similar reasons as the ones explained for the flux quantization in superconducting rings. The lines arrange according to a vortex lattice: the Abrikosov lattice, and translational symmetry gets broken! While the lattice has hexagonal symmetry close to $H_{c 1}$, it may get distorted at intermediate fields up to $H_{c 2}$ in non-cubic systems, when the gradient term in the GL free energy (2.7) is not isotropic. Several techniques have been used to determine the symmetry of the vortex lattice and relate it with the anisotropies in the system (decoration with magnetic particles, neutron scattering) [8].

Higgs-Anderson mechanism. Let us mention another symmetry-related aspect of the GL theory. At temperatures well below the critical temperature, the modulus of the order parameter is fixed and the free energy (2.7) reduces to:

$$
F[\theta, \boldsymbol{A}]=F_{s}+\int d \boldsymbol{x}\left[\frac{\hbar^{2} n_{s}}{8 m}\left(\nabla \theta-\frac{2 e}{\hbar c} \boldsymbol{A}\right)^{2}+\frac{1}{8 \pi}(\operatorname{rot} \boldsymbol{A})^{2}\right] .
$$

a cylindrical geometry with external magnetic field applied along the cylinder axis $\hat{z}$, the relevant free energy is obtained after a Legendre transformation, $\tilde{F}=F-\int d \boldsymbol{x}\left(\boldsymbol{h}_{\text {ext }} \cdot \boldsymbol{h} / 4 \pi\right)$ (see Ref. [7] for details). In the normal state, one then inserts $\boldsymbol{h}_{\text {ext }}=\boldsymbol{h}=H_{c} \hat{z}$ in the expression for the free energy and obtains the result for the magnetic energy given above. Instead, $\boldsymbol{h}=0$ in the Meissner state and such a term does not appear.

4 This equation reads:

$$
-\frac{\hbar^{2}}{2 m} \frac{\partial^{2} \psi}{\partial x^{2}}+\frac{1}{2} m \omega^{2} x^{2} \psi(x)=E \psi(x)
$$

where $m$ is the particle's mass and $\omega / 2 \pi$ is the oscillator's frequency. The eigenenergies of this equation are $E_{n}=(n+1 / 2) \hbar \omega$ at integer $n \geq 0$

${ }^{5}$ We also mention without derivation that $H_{c 1}=H_{c}(\ln \kappa / \kappa \sqrt{2})<H_{c}$ at large $\kappa$, while $H_{c 1}=H_{c}=H_{c 2}$ at $\kappa=1 / \sqrt{2}$. 


\section{Contribution of Symmetries in Condensed Matter}

Here, $F_{s}$ is the free energy in a uniform superconducting state in the absence of magnetic field, while the other terms originate from the variations of the phase of the order parameter and the vector potential. If the order parameter did not couple to the electromagnetic field, the free energy would only retain a term $F_{\text {kin }} \propto \int d \boldsymbol{x}(\nabla \theta)^{2}$ or, equivalently in Fourier space, $F_{\text {kin }} \propto \sum_{\boldsymbol{q}} \boldsymbol{q}^{2}\left|\theta_{\boldsymbol{q}}\right|^{2}$. Such a term vanishes in the long-wavelength limit, $\boldsymbol{q} \rightarrow 0$. This is characteristic of the Goldstone soft mode that manifests in second order phase transitions and states that smooth variations of the order parameter cost little energy. Actually, this kinetic term is at the origin of the superfluidity: for instance, it is sufficient to describe the properties of superfluid ${ }^{4} \mathrm{He}$. However, in the superconducting case, the order parameter is related with the electrons that carry a charge, thus it also couples to $\boldsymbol{A}$. Then, let us derive from Eq. (2.21) a free energy that only depends on $\boldsymbol{A}$, so that

$$
e^{-F[\boldsymbol{A}] / k_{B} T}=\int \mathcal{D} \theta e^{-F[\theta, \boldsymbol{A}] / k_{B} T}
$$

Here, $\mathcal{D} \theta$ is a normalized measure of integration performed on all the realizations of the function $\theta(\boldsymbol{x})$. In Fourier space, we introduce longitudinal and transversal components of $\boldsymbol{A}_{\boldsymbol{q}}$, with respect to $\boldsymbol{q}$ :

$$
A_{q}=A_{q}^{\|}+A_{q}^{\perp}
$$

so that

$$
\boldsymbol{A}_{\boldsymbol{q}}^{\|}=\frac{\left(\boldsymbol{q} \cdot \boldsymbol{A}_{\boldsymbol{q}}\right) \boldsymbol{q}}{q^{2}} \quad \text { and } \quad \boldsymbol{A}_{\boldsymbol{q}}^{\perp}=\boldsymbol{A}_{\boldsymbol{q}}-\frac{\left(\boldsymbol{q} \cdot \boldsymbol{A}_{\boldsymbol{q}}\right) \boldsymbol{q}}{q^{2}} .
$$

Then, Eq. (2.21) can be expressed as

$$
\begin{aligned}
F=F_{S}+\sum_{\boldsymbol{q}} & {\left[\frac{\hbar^{2} n_{s}}{8 m}\left(i \boldsymbol{q} \theta_{\boldsymbol{q}}-\frac{2 e}{\hbar c} \boldsymbol{A}_{\boldsymbol{q}}\right) \cdot\left(-i \boldsymbol{q} \theta_{-\boldsymbol{q}}-\frac{2 e}{\hbar c} \boldsymbol{A}_{-\boldsymbol{q}}\right)+\frac{1}{8 \pi}\left(i \boldsymbol{q} \times \boldsymbol{A}_{\boldsymbol{q}}\right) \cdot\left(-i \boldsymbol{q} \times \boldsymbol{A}_{-\boldsymbol{q}}\right)\right] } \\
=F_{S}+\sum_{\boldsymbol{q}} & {\left[\frac{\hbar^{2} n_{s} q^{2}}{8 m} \tilde{\theta}_{\boldsymbol{q}} \tilde{\theta}_{-\boldsymbol{q}}+\frac{n_{s} e^{2}}{2 m c^{2}}\left(\boldsymbol{A}_{\boldsymbol{q}} \cdot \boldsymbol{A}_{-\boldsymbol{q}}-\frac{1}{q^{2}}\left(\boldsymbol{q} \cdot \boldsymbol{A}_{\boldsymbol{q}}\right) \cdot\left(\boldsymbol{q} \cdot \boldsymbol{A}_{-\boldsymbol{q}}\right)\right)\right.} \\
& \left.+\frac{1}{8 \pi}\left(\boldsymbol{q} \times \boldsymbol{A}_{\boldsymbol{q}}^{\perp}\right) \cdot\left(\boldsymbol{q} \times \boldsymbol{A}_{-\boldsymbol{q}}^{\perp}\right)\right],
\end{aligned}
$$

with $\tilde{\theta}_{\boldsymbol{q}}=\theta_{\boldsymbol{q}}+\left(2 e i / \hbar c q^{2}\right) \boldsymbol{q} \cdot \boldsymbol{A}_{\boldsymbol{q}}$. The Gaussian integration over $\theta_{\boldsymbol{q}}$ in Eq. (2.22) is now readily performed. The resulting free energy is

$$
F[\boldsymbol{A}]=F_{S}+\frac{1}{8 \pi} \sum_{\boldsymbol{q}}\left(q^{2}+\frac{1}{\lambda_{L}^{2}}\right) \boldsymbol{A}_{\boldsymbol{q}}^{\perp} \cdot \boldsymbol{A}_{-\boldsymbol{q}}^{\perp} .
$$

Equation (2.26) is yet another way to write the London free energy (2.1). It has the advantage to be an electromagnetic-gauge invariant function of the vector potential. The term $\propto q^{2}$ corresponds to the usual magnetic energy, while the second term exists only in the superconducting state. In vacuum, the first term corresponds to photons with energy $E=q c$. A way to interpret the second term in Eq. (2.26) is to state that photons have acquired a mass, like in the Einstein equation for massive particles $E^{2}=q^{2} c^{2}+m^{2} c^{4}$. This mechanism of mass generation by "exhaustion" of the Goldstone mode $\theta$ was noticed by several researchers, in particular by Anderson in 1958 and Higgs in 1964. Its most important application was in the field of particle physics where it is now the most commonly believed scenario to explain the mass of quarks and leptons. Indeed, the electroweak theory by Salam and Weinberg corresponds to fundamental particles with zero mass, that are described by gauge fields analogous to $\boldsymbol{A}$. Then, it was postulated that such particles couple to a bosonic field (the Higgs field) with a Goldstone mode, like the phase of the superconducting order parameter $\eta$, the exhaustion of which leads to the mass generation of leptons. The observation of the Higgs boson is one of the main goals of modern research in particle physics. 


\section{EPJ Web of Conferences}

\subsection{Josephson effect - measuring the phase of the order parameter}

We already mentioned that order parameters with the same amplitude but different phases correspond to different superconducting states. Actually, the phase of the order parameter is (in some sense) a measurable quantity as we explain now. Indeed, let us consider two superconductors separated by a thin insulating barrier, each of them filling a half-space. In the normal state, quantum mechanics allows electrons getting through the barrier by the tunnel effect. Thus, it is also reasonable to assume that the order parameters in each superconductor get coupled. That is, the free energy describing the whole system,

$$
F=F_{1}+F_{2}+F_{12}
$$

where

$$
F_{i}=F_{n i}+\int_{V_{i}} d \boldsymbol{x}\left[\alpha_{i}\left|\eta_{i}\right|^{2}+\frac{\beta_{i}}{2}\left|\eta_{i}\right|^{4}+K_{i}\left|\nabla \eta_{i}\right|^{2}\right]
$$

are the free energies (at zero magnetic field) for each superconductor filling volume $V_{i}(i=1,2)$ and with order parameter $\eta_{i}$, also contains a boundary energy term,

$$
F_{12}=\gamma \int d \boldsymbol{x}\left[\eta_{1}^{*} \eta_{2}+\eta_{2}^{*} \eta_{1}\right] \delta(x)
$$

that fulfills the required global $U(1)$ gauge symmetry, with $\eta_{i} \rightarrow \eta_{i} e^{i \theta_{0}}$, as well.

Minimizing the total free energy with respect to $\eta_{1}^{*}$ and $\eta_{2}^{*}$, we find that the orders parameters obey the GL equations (2.10) inside each superconductor and

$$
\begin{aligned}
& \left.K_{1} \frac{\partial \eta_{1}}{\partial x}\right|_{x=0}=-\gamma \eta_{2}(0), \\
& \left.K_{2} \frac{\partial \eta_{2}}{\partial x}\right|_{x=0}=\gamma \eta_{1}(0),
\end{aligned}
$$

at their interface $(x=0)$. We further assume that $\gamma$ is small so that, inside each superconductor, the order parameter is approximately constant and of the form $\eta_{i}=\left|\eta_{i}\right| e^{i \theta_{i}}$. The current flowing through the interface is then evaluated at $x \rightarrow 0^{-}$in the first order in $\gamma$ by inserting Eq. (2.30a) into (2.12). As a result, we find the "first" Josephson relation

$$
I=I_{c} \sin \theta,
$$

where $\theta=\theta_{1}-\theta_{2}$ is the phase difference. That is, the junction between two superconductors can sustain a dissipationless DC current with maximal amplitude

$$
I_{c}=(4 e / \hbar) \gamma S\left|\eta_{1}\right|\left|\eta_{2}\right|,
$$

where $S$ is the junction area. Such a device is called a Josephson junction and $I_{c}$ is its critical current. Measuring the current in the dissipationless regime is then analogous to measuring the superconducting phase difference.

The discussion can be extended to the case of voltage biased junctions by invoking the electromagnetic gauge invariance. Indeed, we already mentioned that the gauge transformation $\boldsymbol{A} \rightarrow$ $\boldsymbol{A}+\nabla \chi$ should be supplemented with $\eta \rightarrow \eta \exp (2 i e \chi / \hbar c)$ so that the GL functional remains invariant. Simultaneously, the electric potential should be transformed according to $V \rightarrow V-(1 / c) \partial \chi / \partial t$. Thus, the phase difference and bias voltage are related by the "second" Josephson relation:

$$
\frac{\partial \theta}{\partial t}=\frac{2 e V}{\hbar} .
$$




\section{Contribution of Symmetries in Condensed Matter}

We solve Eq. (2.33) for the phase at fixed voltage, insert the result into Eq. (2.31), and find that the current through a voltage-biased Josephson junction oscillates like

$$
I=I_{c} \sin \left(\frac{2 e V t}{\hbar}\right) .
$$

This is called the AC Josephson effect.

The predictions of both the DC and AC Josephson effects, as well as the microscopic theory of these effects, were done by Josephson in 1962.

\section{MICROSCOPIC THEORY - CONVENTIONAL AND UNCONVENTIONAL SUPERCONDUCTIVITY}

In this Section, we introduce the basics of the microscopic theory of superconductivity that allow giving a deeper meaning to the superconducting order parameter. Consequently, it is possible to discuss more complex scenarios for symmetry breaking that can take place at the superconducting transition. Conventional superconductors - with only $U(1)$ gauge symmetry breaking - and unconventional superconductors - with additional symmetry breaking - are contrasted, and their distinct physical properties are discussed.

Such theories based on symmetry arguments had been primarily developed to account for the properties of superfluid Helium 3 [9]. They have been crucial in understanding several properties of the heavy-fermion, organics and high-temperature superconductors discovered since the late 70's, in strong contrast with those known in usual superconductors, even if the precise microscopic theory of superconductivity in these systems remains elusive.

\subsection{Cooper instability in isotropic Fermi liquid}

We will now show from a very simplified model that a metal is unstable in the presence of a weak attractive interaction between the electrons. This so-called Cooper instability was the first step toward the microscopic theory of superconductivity formulated by Bardeen, Cooper, and Schrieffer (BCS) in 1957.

In the absence of interaction, a metal is most simply described with an isotropic Fermi gas model. In this model, an electron with momentum $\boldsymbol{k}$ has a kinetic energy $\varepsilon_{k}=\hbar^{2} k^{2} / 2 m$ where $k=|\boldsymbol{k}|$ is the modulus of $\boldsymbol{k}$ and $m$ is the electron's mass. In the ground state, the electronic states with energy below the Fermi energy are doubly occupied with electrons of opposite spins, while the states above the Fermi level are empty. The ground state is also called the Fermi sea.

We now assume that two electrons at positions $\boldsymbol{x}_{1}$ and $\boldsymbol{x}_{2}$ interact through a potential $V\left(\boldsymbol{x}_{1}, \boldsymbol{x}_{2}\right)=$ $V\left(\left|x_{1}-x_{2}\right|\right)$ that only depends on their relative distance. To demonstrate the Cooper instability, we consider a variational wavefunction $\psi_{\sigma_{1} \sigma_{2}}\left(\boldsymbol{x}_{1}, \boldsymbol{x}_{2}\right)$ for two electrons with spins $\sigma_{1}$ and $\sigma_{2}$ along the spin quantization axis and residing above the Fermi sea. The energy $E$ of this state, counted from the Fermi energy $\varepsilon_{F}$, is obtained by solving the Schrödinger equation

$$
\left[-\frac{\hbar^{2}}{2 m}\left(\nabla_{1}^{2}+\nabla_{2}^{2}\right)-2 \varepsilon_{F}+V\left(\boldsymbol{x}_{1}, \boldsymbol{x}_{2}\right)\right] \psi_{\sigma_{1} \sigma_{2}}\left(\boldsymbol{x}_{1}, \boldsymbol{x}_{2}\right)=E \psi_{\sigma_{1} \sigma_{2}}\left(\boldsymbol{x}_{1}, \boldsymbol{x}_{2}\right) .
$$

As the interaction potential does not depend on the spin, the wavefunction can be sought in a separable form: $\psi_{\sigma_{1} \sigma_{2}}\left(\boldsymbol{x}_{1}, \boldsymbol{x}_{2}\right)=\psi\left(\boldsymbol{x}_{1}, \boldsymbol{x}_{2}\right) \chi_{\sigma_{1} \sigma_{2}}$.

We look for a solution of Eq. (3.1) with the electrons' center of motion at rest:

$$
\psi\left(\boldsymbol{x}_{1}, \boldsymbol{x}_{2}\right)=\psi\left(\boldsymbol{x}_{1}-\boldsymbol{x}_{2}\right)=\sum_{\boldsymbol{k}} \psi(\boldsymbol{k}) e^{i \boldsymbol{k} \cdot\left(\boldsymbol{x}_{1}-\boldsymbol{x}_{2}\right)}
$$




\section{EPJ Web of Conferences}

That is, electrons in the pair have opposite momenta $\boldsymbol{k}_{1}=-\boldsymbol{k}_{2} \equiv \boldsymbol{k}$. As they live above the Fermi surface, the sum is restricted to $|\boldsymbol{k}|>k_{F}$, where $k_{F}$ is the Fermi momentum $\left(\varepsilon_{F}=\hbar^{2} k_{F}^{2} / 2 m\right)$. We also define

$$
V_{\boldsymbol{k}_{1} \boldsymbol{k}_{2}}=\int d \boldsymbol{x} e^{i\left(\boldsymbol{k}_{1}-\boldsymbol{k}_{2}\right) \cdot \boldsymbol{x}} V(|\boldsymbol{x}|)
$$

Then, Eq. (3.1) yields

$$
\left(2 \xi_{k_{1}}-E\right) \psi\left(\boldsymbol{k}_{1}\right)+\sum_{\boldsymbol{k}_{2}} V_{\boldsymbol{k}_{1} \boldsymbol{k}_{2}} \psi\left(\boldsymbol{k}_{2}\right)=0
$$

where $\xi_{k}=\varepsilon_{k}-\varepsilon_{F}$.

Now we note that, according to Eq. (3.3), $V_{\boldsymbol{k}_{1} \boldsymbol{k}_{2}}$ only depends on $\left|\boldsymbol{k}_{1}-\boldsymbol{k}_{2}\right|=\left(k_{1}^{2}+k_{2}^{2}-\right.$ $\left.2 k_{1} k_{2} \cos \theta\right)^{1 / 2}$, that is on $k_{1}, k_{2}$ and the relative angle $\theta$ between the directions $\hat{\boldsymbol{k}}_{1}$ and $\hat{\boldsymbol{k}}_{2}$ of $\boldsymbol{k}_{1}$ and $\boldsymbol{k}_{2}$. Therefore it can be decomposed into the following form:

$$
V_{\boldsymbol{k}_{1} \boldsymbol{k}_{2}}=\sum_{l=0}^{\infty}(2 l+1) V_{l}\left(k_{1}, k_{2}\right) P_{l}(\cos \theta),
$$

where $P_{l}(\cos \theta)$ are the Legendre polynomials.

We look for a solution of Eq. (3.4) in the form $\psi(\boldsymbol{k})=\phi_{l}(k) Y_{l m}(\hat{\boldsymbol{k}})$, where $Y_{l m}(\hat{\boldsymbol{k}})$ are the spherical harmonics for a given angular momentum $l$ and a projection $m$ along the quantization axis. Using the identity

$$
P_{l}(\cos \theta)=\frac{4 \pi}{2 l+1} \sum_{m=-l}^{l} Y_{l m}\left(\hat{\boldsymbol{k}}_{2}\right)^{*} Y_{l m}\left(\hat{\boldsymbol{k}}_{1}\right)
$$

and the relation $\int d \Omega_{\boldsymbol{k}} Y_{l m}(\hat{\boldsymbol{k}})^{*} Y_{l^{\prime} m^{\prime}}(\hat{\boldsymbol{k}})=\delta_{l l^{\prime}} \delta_{m m^{\prime}},{ }^{6}$ we transform Eq. (3.4) into

$$
\left(2 \xi_{k_{1}}-E\right) \phi_{l}\left(k_{1}\right)=-\frac{1}{2 \pi^{2}} \int_{k_{F}}^{\infty} d k_{2} k_{2}^{2} V_{l}\left(k_{1}, k_{2}\right) \phi_{l}\left(k_{2}\right) .
$$

In general, the detailed behavior of the interaction potential is determined by many contributions including electron-electron and electron-phonon interactions. As a very crude simplification, we assume that the electrons interact attractively with a constant interaction, in the channel with orbital momentum $l$, and in an energy bandwidth $2 \hbar \Omega$ :

$$
V_{l}\left(k_{1}, k_{2}\right)= \begin{cases}-\left|V_{l}\right| & \text { at }\left|\xi_{k_{1}}\right|,\left|\xi_{k_{2}}\right|<\hbar \Omega, \\ 0 & \text { otherwise. }\end{cases}
$$

Assuming $\hbar \Omega \ll \varepsilon_{F}$, we can linearize the electron spectrum in vicinity of the Fermi level and, then, rewrite Eq. (3.7) as

$$
\phi_{l}\left(k_{1}\right)=\frac{v_{0}\left|V_{l}\right|}{\left(2 \xi_{k_{1}}-E\right)} \int_{0}^{\hbar \Omega} d \xi_{k_{2}} \phi_{l}\left(k_{2}\right),
$$

where $v_{0}=m k_{F} /\left(2 \pi^{2} \hbar^{2}\right)$ is the electronic density of states per spin at the Fermi level. The wavefunctions can be eliminated from Eq. (3.9) after integrating both sides over $\xi_{k_{1}}$. We then obtain the equation determining the eigenenergy of the electron pair in Eq. (3.1):

$$
1=\int_{0}^{\hbar \Omega} d \xi_{k} \frac{v_{0}\left|V_{l}\right|}{\left(2 \xi_{k}-E\right)} \approx \frac{v_{0}\left|V_{l}\right|}{2} \ln \left(\frac{2 \hbar \Omega}{-E}\right) .
$$

\footnotetext{
${ }^{6}$ We also take the continuum limit with $\sum_{k} \rightarrow \int d \Omega_{\hat{k}} \int k^{2} d k /(2 \pi)^{3}$.
} 


\section{Contribution of Symmetries in Condensed Matter}

It admits a solution with negative energy,

$$
E=-2 \hbar \Omega \exp \left(-\frac{2}{v_{0}\left|V_{l}\right|}\right)
$$

We discuss the implications of this result below.

Equation (3.11) first demonstrates that the Fermi surface in the normal state is unstable. Indeed, we obtained a variational wavefunction composed of two particles taken above the Fermi sea, with negative energy measured from the Fermi level. This is in contradiction with the fact that the Fermi sea was initially assumed to be the ground state. Moreover, we observe that this instability takes place at arbitrarily small value of the attractive potential, $\left|V_{l}\right|$, and that the energy $E$ depends non-analytically on $\left|V_{l}\right|$. This explains that the theory of superconductivity cannot be elaborated by a standard perturbative approach in the interaction parameter $V_{l}$, even when it is small, as all orders in the perturbation are important for the result.

Nevertheless, this calculation already contains the idea that electron pairs form a bound state (the Cooper pair), with a binding energy $\Delta \sim|E|$. While the electrons are fermions, the Cooper pairs are bosons. Thus, they can be subject to a Bose-Einstein condensation, thereby explaining the superfluid properties of the electrons. Both the Cooper pair formation and their Bose-Einstein condensation take place at the same critical temperature, estimated as $T_{c} \sim \Delta / k_{B}$. Note however that the typical interelectronic distance in metals is of the order of the Fermi wavelength, while the size of the Cooper pairs, roughly estimated as $\xi \sim \hbar v_{F} / \Delta$ where $v_{F}$ is the Fermi velocity, is typically much larger (10$100 \mathrm{~nm}$ ). Thus the pairs strongly overlap. In practice, the theory of superconductivity is not a two-body problem as assumed above, but a many-body problem for the solution of which an advanced quantumstatistical field theory needs being introduced. A hint to such a theory will be given in Sec. 3.4.

Assuming that $T_{c}$ depends on the interaction parameter like in Eq. (3.11), we deduce that only the most attractive channel determines the superconducting properties. Indeed it is unlikely for two critical temperatures in different channels $l, l^{\prime}$ to be accidentally close to each other. Note also that at finite $l \leq 1$, the Cooper pairs may carry an orbital momentum depending on their spherical harmonic content. Therefore, the superconducting state can display an orbital magnetism under some circumstances. This is only one of the differences between the trivial pairing, at $l=0$, and non-trivial pairings, at $l \neq 0$, to be discussed in more details below.

\subsection{Superconducting order parameter and spin structure of the Cooper pair}

The above calculation justifies introducing the superconducting order parameter as the Cooper pair wavefunction in Fourier space, $\psi_{\sigma_{1} \sigma_{2}}(\hat{\boldsymbol{k}})$. (Electrons are taken close to the Fermi surface, with fixed $k \simeq k_{F}$.) We now discuss further constraints on the structure of the order parameter, as well as some consequences of it.

The wavefunction is composed of fermionic particles. Thus, it must be antisymmetric under their permutations: $\psi_{\sigma_{2} \sigma_{1}}(-\hat{\boldsymbol{k}})=-\psi_{\sigma_{1} \sigma_{2}}(\hat{\boldsymbol{k}})$. Within the isotropic model of the previous section, we found that superconductivity takes place in channel $l$, with $\psi_{\sigma_{1} \sigma_{2}}(\hat{\boldsymbol{k}}) \propto Y_{l m}(\hat{\boldsymbol{k}}) \chi_{\sigma_{1} \sigma_{2}}$. Thus, we deduce that at even orbital momentum, $l=0,2, \ldots$, the spin component is odd: $\chi_{\sigma_{1} \sigma_{2}}=-\chi_{\sigma_{2} \sigma_{1}}$. For two spin- $\frac{1}{2}$ electrons, this means that $\chi$ is the spin wavefunction for the singlet state with total spin $S=0, \chi=|\uparrow \downarrow>-| \downarrow \uparrow>$. However, for odd $l=1,3, \ldots$, the spin component is even; it corresponds to triplet states with total spin $S=1$ and projection along the quantization axis $M=1,0$, or -1 , with $\chi=|\uparrow \uparrow>,| \uparrow \downarrow>+\mid \downarrow \uparrow>$, or $\mid \downarrow \downarrow>$, respectively.

It is convenient to introduce $2 \times 2$ Pauli matrices

$$
\sigma_{0}=\left(\begin{array}{ll}
1 & 0 \\
0 & 1
\end{array}\right), \quad \sigma_{x}=\left(\begin{array}{ll}
0 & 1 \\
1 & 0
\end{array}\right), \quad \sigma_{y}=\left(\begin{array}{cc}
0 & -i \\
i & 0
\end{array}\right), \quad \text { and } \quad \sigma_{z}=\left(\begin{array}{cc}
1 & 0 \\
0 & -1
\end{array}\right)
$$




\section{EPJ Web of Conferences}

with horizontal (respectively vertical) entries corresponding to the spin state of electron 1 (2). Then, the order parameter can be written as a matrix in spin space. In the singlet state, it reads:

$$
\hat{\psi}(\hat{\boldsymbol{k}})=g(\hat{\boldsymbol{k}}) i \sigma_{y},
$$

where $g(\hat{\boldsymbol{k}})$ is a scalar, even function of $\hat{\boldsymbol{k}}$. Singlet Cooper pairs don't carry a spin, but they may carry an orbital momentum:

$$
\boldsymbol{L}=i \hbar \int \frac{d \Omega_{\hat{\boldsymbol{k}}}}{4 \pi} g(\hat{\boldsymbol{k}})^{*}\left(\frac{\partial}{\partial \boldsymbol{k}} \times \boldsymbol{k}\right) g(\hat{\boldsymbol{k}}) .
$$

In the triplet state, the order parameter reads

$$
\hat{\psi}(\hat{\boldsymbol{k}})=[\boldsymbol{d}(\hat{\boldsymbol{k}}) \cdot \boldsymbol{\sigma}] i \sigma_{y},
$$

where $\boldsymbol{\sigma}=\left(\sigma_{x}, \sigma_{y}, \sigma_{z}\right)$ and the order parameter $\boldsymbol{d}(\hat{\boldsymbol{k}})=\left(d_{x}(\hat{\boldsymbol{k}}), d_{y}(\hat{\boldsymbol{k}}), d_{z}(\hat{\boldsymbol{k}})\right)$ is an odd vector function so that $-d_{x}+i d_{y}, d_{z}$, and $d_{x}+i d_{y}$ are the amplitudes of the triplet components with $M=1,0$, and -1 , respectively. The spin carried by the Cooper pairs along $\hat{z}$-axis is readily found: $S_{z}=$ $(-i \hbar) \int\left(d \Omega_{\hat{\boldsymbol{k}}} / 4 \pi\right)\left[d_{x}(\hat{\boldsymbol{k}})^{*} d_{y}(\hat{\boldsymbol{k}})-d_{y}(\hat{\boldsymbol{k}})^{*} d_{x}(\hat{\boldsymbol{k}})\right]$. This relation generalizes into

$$
\boldsymbol{S}=(-2 i \hbar) \int \frac{d \Omega_{\hat{\boldsymbol{k}}}}{4 \pi} \boldsymbol{d}(\hat{\boldsymbol{k}})^{*} \times \boldsymbol{d}(\hat{\boldsymbol{k}}) .
$$

We note that Eq. (3.16) vanishes if $\boldsymbol{d}(\hat{\boldsymbol{k}})$ is proportional to a real vector. The orbital momentum of the Cooper pairs is

$$
\boldsymbol{L}=i \hbar \sum_{i} \int \frac{d \Omega_{\hat{\boldsymbol{k}}}}{4 \pi} d_{i}(\hat{\boldsymbol{k}})^{*}\left(\frac{\partial}{\partial \boldsymbol{k}} \times \boldsymbol{k}\right) d_{i}(\hat{\boldsymbol{k}}) .
$$

We can already expect strong differences between singlet and triplet superconductivity. In singlet superconductors, the Cooper pairs are formed with electrons in time-reversed states, $\mid \boldsymbol{k} \uparrow>$ and $\mid-\boldsymbol{k} \downarrow>$. Both electrons should reside in vicinity of the Fermi surface, $\xi_{\boldsymbol{k} \uparrow}=\xi_{-\boldsymbol{k} \downarrow}$. Thus, singlet superconductivity is destroyed by magnetic effects (Zeeman effect of the magnetic field, ferromagnetism, magnetic impurities) that will break this energy degeneracy.

Instead, triplet superconductors involve Cooper pairs where electrons are only required to have opposite momenta. Thus, ferromagnetic superconductors are most likely to be of the triplet kind, with spin magnetism (Cooper pairs with electrons of the same spin).

\subsection{Superconducting phases in crystals}

Having established the structure of the order parameter, we can now discuss the superconducting transition as a spontaneous symmetry breaking in more details than in Section 2.

According to the Landau theory of phase transitions, the normal phase at $T>T_{c}$ contains all the symmetries of the system. The symmetry group is $G=\mathcal{G} \times \mathcal{R} \times U(1)$. Here, $\mathcal{G}$ is the symmetry group of the crystal together with the group of spin rotations, and $\mathcal{R}$ is the time-reversal symmetry. At $T<T_{c}$, the possible superconducting states are classified by the subgroups $H \subset G$ that leave the superconducting order parameter, $\hat{\psi}(\hat{\boldsymbol{k}})$, invariant. The enumeration of all superconducting classes for a given crystal is similar to the construction of the magnetic classes. The superconducting classes at the transition are in one-to-one correspondence with the irreducible representations (IRs) $\Gamma$ of $G$. An IR can be represented with a set of basis functions $\psi_{i}^{\Gamma}(\hat{\boldsymbol{k}})$, for $1 \leq i \leq d_{\Gamma}$, where $d_{\Gamma}$ is the IR's dimension, so that any function within this set is transformed into a linear combination of all the other ones under the symmetry operations $g$ belonging to $G$. An attractive interaction parameter $\left|V_{\Gamma}\right|$ is indeed a property of the IR and a critical temperature $T_{c}(\Gamma)$ can then be attributed to it. Due to the exponential dependence of $T_{c}(\Gamma)$ with $\left|V_{\Gamma}\right|$, similar to Eq. (3.11), it is unlikely that two IRs have accidentally close critical temperatures. Therefore, the superconducting transition is specific to one of the IRs of $G$, only. 


\section{Contribution of Symmetries in Condensed Matter}

Let us illustrate this vocabulary with two specific examples:

- In Sec. 2, we considered that only the $U(1)$ gauge symmetry was broken. Thus, $H=\mathcal{G} \times R$. The corresponding IR is denoted $A_{1 g}$, it is one-dimensional; the basis function can be taken as a constant, $\psi^{A_{1 g}}(\hat{\boldsymbol{k}})=1$. This corresponds to "conventional" superconductivity.

- In the discussion on the Cooper instability in Sec. 3.1, we encountered the IRs of the group of rotations $S O(3)$ for an isotropic medium. They are labelled with the angular momentum $l$, have dimension $2 l+1$, and the spherical harmonics $Y_{l m}$, with $|m| \leq l$, form a set of basis functions. The "conventional case" corresponds to $l=0$. The atomic nomenclature is sometimes used and states with $l=0,1,2, \ldots$ are called s-wave (the conventional state), $\mathrm{p}$-wave, $\mathrm{d}$-wave, $\ldots$ states.

More generally, "unconventional" superconductivity is realized as soon as $H \neq \mathcal{G} \times R$. The aim of the rest of the notes is to discuss the differences between conventional and unconventional superconductivity, among which the possibility for spin and/or orbital magnetism in the unconventional case was already mentioned.

To simplify the discussion, we make two assumptions. We first assume that only the point group of the crystal needs being considered. We also consider the case when the crystal structure contains an inversion center that is conserved below $T_{c}$, so that

$$
I \hat{\psi}(\hat{\boldsymbol{k}})=\hat{\psi}(-\hat{\boldsymbol{k}})= \pm \hat{\psi}(\hat{\boldsymbol{k}})
$$

Here, $I$ is the inversion operator. The orbital part of the order parameter is thus necessarily either even or odd. As the order parameter is also antisymmetric, the spin content must be either singlet (in the even case) or triplet (in the odd case): there is no singlet-triplet mixing. In the singlet states, the order parameter reads:

$$
g(\hat{\boldsymbol{k}})=\sum_{i=1}^{d_{\Gamma}} \eta_{i} \psi_{i}^{\Gamma}(\hat{\boldsymbol{k}}) .
$$

Here, $\eta_{i}$ are the complex amplitudes for the components of the order parameter. In triplet superconductors, there is a distinction between crystals with weak spin-orbit interaction, where

$$
\boldsymbol{d}(\hat{\boldsymbol{k}})=\sum_{i=1}^{d_{\Gamma}} \boldsymbol{\eta}_{i} \psi_{i}^{\Gamma}(\hat{\boldsymbol{k}})
$$

and $\boldsymbol{\eta}_{i}=\left(\eta_{i x}, \eta_{i y}, \eta_{i z}\right)$ has three components (the order parameter has $3 d_{\Gamma}$ components), and crystals with strong spin-orbit interaction, ${ }^{7}$ where the spin components of the basis vector-functions, $\psi_{i}^{\Gamma}=$ $\left(\psi_{i x}^{\Gamma}, \psi_{i y}^{\Gamma}, \psi_{i z}^{\Gamma}\right)$, are rigidly fixed with the crystal axes and

$$
\boldsymbol{d}(\hat{\boldsymbol{k}})=\sum_{i=1}^{d_{\Gamma}} \eta_{i} \psi_{i}^{\Gamma}(\hat{\boldsymbol{k}}) .
$$

The order parameter $\eta_{i}$ has then $d_{\Gamma}$ components. Note that the superconducting order parameter in the singlet case or in the triplet case with strong spin-orbit coupling is one-component if $d_{\Gamma}=1$. Then, the superconducting class is in one-to-one correspondence with the IR. Otherwise, the order parameter is multicomponent and several superconducting states with the same critical temperature but with different free energies below the superconducting transition can exist.

Let us consider two examples.

\footnotetext{
7 Although the spin is no more a good quantum number in the presence of spin-orbit coupling, the Kramers degeneracy due to time-reversal symmetry ensures that electronic states are doubly degenerate. They can thus be classified according to a "pseudospin" to which the above discussion refers.
} 


\section{EPJ Web of Conferences}

Superfluid Helium 3. This isotope of Helium undergoes a superfluid transition at low pressure $(<34$ bars) and temperatures (in the $\mathrm{mK}$ range). This transition is very different from the one in ${ }^{4} \mathrm{He}$. Indeed, the ${ }^{3} \mathrm{He}$ nucleus is composed of two protons and one neutron. Thus, it is a fermion. The origin of its superfluidity is attributed to a Cooper pairing of ${ }^{3} \mathrm{He}$ atoms.

In the normal phase at low pressure, ${ }^{3} \mathrm{He}$ is a fluid. The superfluid classes are thus classified according to the IRs of $S O(3)$. A strong hard-core repulsion between the atoms prevents their pairing in $l=0$ state. ${ }^{3} \mathrm{He}$ was soon realized to be a superfluid of the p-wave $(l=1)$, triplet kind, with a 9component order parameter. As

$$
Y_{11}(\hat{\boldsymbol{k}}) \sim \hat{k}_{x}-i \hat{k}_{y}, \quad Y_{10}(\hat{\boldsymbol{k}}) \sim \hat{k}_{z}, \quad \text { and } \quad Y_{1-1}(\hat{\boldsymbol{k}}) \sim \hat{k}_{x}+i \hat{k}_{y},
$$

the order parameter $d_{\alpha}(\hat{\boldsymbol{k}})=\sum_{m=-1}^{1} \eta_{\alpha m} Y_{1 m}(\hat{\boldsymbol{k}})$, at $\alpha=x, y, z$, can be written alternatively,

$$
d_{\alpha}(\hat{\boldsymbol{k}})=\sum_{i=x, y, z} A_{\alpha i} \hat{k}_{i}
$$

Actually, different superfluid phases were discovered in the phase diagram, depending on the pressure, temperature, and magnetic field; they correspond to different contents in $\left\{A_{\alpha i}\right\}$ :

- The B-phase corresponds to $\boldsymbol{d}(\hat{\boldsymbol{k}})=\hat{\boldsymbol{k}}$, i.e. $A_{\alpha i}=\delta_{\alpha i}$. Writing explicitly the spin content of the order parameter, we get

$$
\psi^{B}(\hat{\boldsymbol{k}}) \propto\left(-\hat{k}_{x}+i \hat{k}_{y}\right)\left|\uparrow \uparrow>+\hat{k}_{z}(|\uparrow \downarrow>+| \downarrow \downarrow>)+\left(\hat{k}_{x}+i \hat{k}_{y}\right)\right| \downarrow \downarrow>.
$$

Thus, this state is not spin-polarized. Moreover, a portion of the spins is in a state with $M=0$ and the spin susceptibility in this phase is smaller than in the normal state.

- The A-phase corresponds to $\boldsymbol{d}(\hat{\boldsymbol{k}})=\left(\hat{k}_{x}+i \hat{k}_{y}, 0,0\right)$ or, equivalently,

$$
\psi^{A}(\hat{\boldsymbol{k}}) \propto\left(\hat{k}_{x}+i \hat{k}_{y}\right)(|\uparrow \uparrow>-| \downarrow \downarrow>) .
$$

It is not spin-polarized and it has the same spin susceptibility as in the normal state. It also bears a large angular momentum.

- At finite magnetic field, it evolves into the $\mathrm{A}_{1}$-phase, with $\boldsymbol{d}(\hat{\boldsymbol{k}})=\left(\hat{k}_{x}+i \hat{k}_{y}\right)(1, i, 0)$ or, equivalently,

$$
\psi^{A_{1}}(\hat{\boldsymbol{k}}) \propto\left(\hat{k}_{x}+i \hat{k}_{y}\right) \mid \uparrow \uparrow>.
$$

It has both spin and orbital magnetic moments.

High-temperature superconductors. The highest superconducting critical temperatures (up to 135 $\mathrm{K}$ ) have been discovered in the cuprate compounds in the '80s. The crystal structure in these materials is schematically built of a stack of $\mathrm{CuO}_{2}$ planes, in which the atoms are arranged in a square lattice. The symmetry is tetragonal and the point group of the crystal,

$$
D_{4 h}=D_{4} \times I,
$$

where $D_{4}=\left\{C_{n}, U_{n}\right\}$ at $n=0,1,2,3$ comprises the rotations $C_{n}$ along $\hat{z}$-axis (perpendicular to the $\mathrm{CuO}_{2}$ planes) with a rotation angle $\pi n / 2$ and the rotations $U_{n}$ by an angle $\pi$ around axes $\hat{x} \cos (\pi n / 4)+$ $\hat{y} \sin (\pi n / 4)$. The superconductivity is known to be singlet. The table of the IRs together with basis functions is given in Table 1.

Experiments have demonstrated that the one-dimensional state corresponding to $\Gamma=B_{1 g}$ is realized below $T_{c}$. The order parameter thus varies like

$$
g(\hat{\boldsymbol{k}}) \propto\left(\hat{k}_{x}^{2}-\hat{k}_{y}^{2}\right)=\cos \left(2 \varphi_{\hat{\boldsymbol{k}}}\right)
$$

where $\varphi_{\hat{\boldsymbol{k}}}$ is the angle between $\hat{x}$ axis and $\hat{\boldsymbol{k}}$ in $(x, y)$-plane. It changes its sign as $\varphi_{\hat{\boldsymbol{k}}}$ is varied, and it has nodes at $\hat{k}_{x}= \pm \hat{k}_{y}$, see Fig. 3 . It is called the d-wave state, because its angular dependence can be accounted with spherical harmonics at $l=2$. Nevertheless, its IR is one-dimensional while the IR 


\section{Contribution of Symmetries in Condensed Matter}

Table 1. Basis functions of even irreducible representations of $D_{4 h}$.

\begin{tabular}{llll}
\hline \hline$d_{\Gamma}$ & $\Gamma$ & $\psi_{i}^{\Gamma}(\hat{\boldsymbol{k}})$ & $H$ \\
\hline 1 & $A_{1 g}$ & $a\left(\hat{k}_{x}^{2}+\hat{k}_{y}^{2}\right)+b \hat{k}_{z}^{2}$ & $D_{4}$ \\
1 & $A_{2 g}$ & $\hat{k}_{x} \hat{k}_{y}\left(\hat{k}_{x}^{2}-\hat{k}_{y}^{2}\right)$ & $D_{4}\left(C_{4}\right)$ \\
1 & $B_{1 g}$ & $\hat{k}_{x}^{2}-\hat{k}_{y}^{2}$ & $D_{4}\left(D_{2}\right)$ \\
1 & $B_{2 g}$ & $\hat{k}_{x} \hat{k}_{y}$ & $D_{4}\left(D_{2}^{\prime}\right)$ \\
2 & $E_{g}$ & $\hat{k}_{x} \hat{k}_{z}, \hat{k}_{y} \hat{k}_{z}$ & \\
\hline \hline
\end{tabular}

Table 2. Basis functions of even irreducible representations of $D_{2 h}$.

\begin{tabular}{llll}
\hline \hline$d_{\Gamma}$ & $\Gamma$ & $\psi_{i}^{\Gamma}(\hat{\boldsymbol{k}})$ & $H$ \\
\hline 1 & $A_{1 g}$ & $a+b\left(\hat{k}_{x}^{2}-\hat{k}_{y}^{2}\right)$ & $D_{2}$ \\
1 & $B_{1 g}$ & $\hat{k}_{x} \hat{k}_{y}$ & $D_{2}\left(C_{2}^{z}\right)$ \\
1 & $B_{2 g}$ & $\hat{k}_{x} \hat{k}_{z}$ & $D_{2}\left(C_{2}^{y}\right)$ \\
1 & $B_{3 g}$ & $\hat{k}_{y} \hat{k}_{z}$ & $D_{2}\left(C_{2}^{x}\right)$ \\
\hline \hline
\end{tabular}

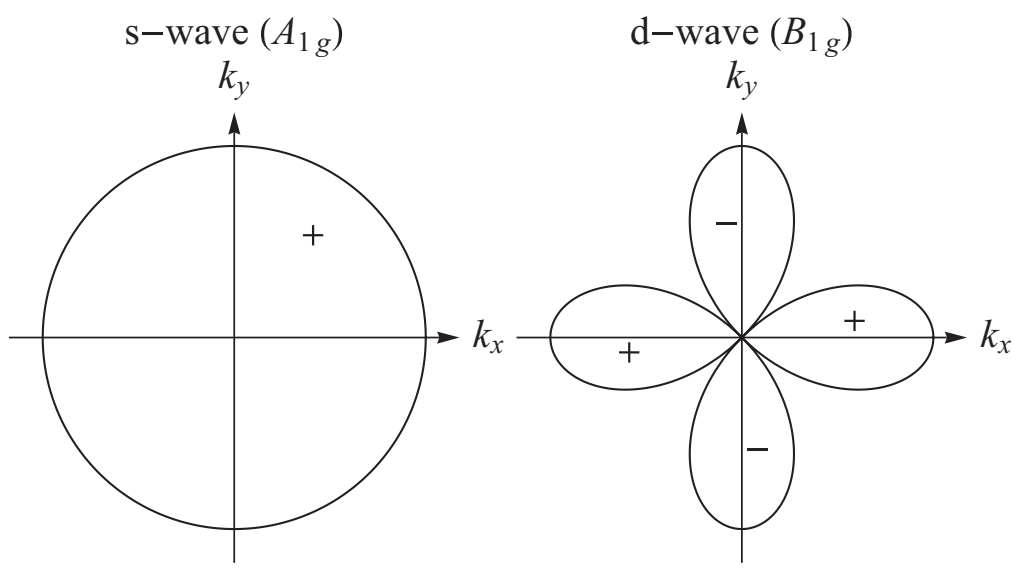

Figure 3. Angular dependence of the order parameter over the Fermi surface in the $\left(k_{x}, k_{y}\right)$-plane for a singlet superconductor with tetragonal symmetry, in the irreducible representation $A_{1 g}$ (s-wave state, left) and $B_{1 g}$ (d-wave state, right). The sign of the order parameter is indicated.

at $l=2$ in isotropic space is 5 -dimensional. The symmetry group in this state is $D_{4}\left(D_{2}\right) \times I$, where $D_{4}\left(D_{2}\right)=\left\{C_{2 k}, U_{2 k}, e^{i \pi} C_{2 k+}, e^{i \pi} C_{2 k+1}\right\}$, at $k=0,1$. The phase $e^{i \pi}=-1$ is needed to compensate the sign change of the order parameter under the rotations.

Let us stress however that symmetries are not always sufficient in determining the superconducting properties. First, the choice of the basis functions is somewhat arbitrary and can only be unambiguously determined with a microscopic model. For instance, one can choose $g(\hat{\boldsymbol{k}}) \propto \cos \left(k_{x} a\right)-\cos \left(k_{y} a\right)$ as well, where $a$ is a lattice constant, to describe the d-wave state. Second, the tetragonal crystal structure may undergo a slight distortion, and become orthorhombic. The symmetry group is then $D_{2 h}$; its IRs are given in Table 2 . We notice that the conventional representation $A_{1 g}$ can be attributed a non-isotropic basis function

$$
g(\hat{\boldsymbol{k}}) \propto a+b \cos \left(2 \varphi_{\hat{\boldsymbol{k}}}\right),
$$

where $a$ and $b$ would depend on the details of a microscopic theory. At $b \neq 0$, Eq. (3.29) describes a conventional, but anisotropic order parameter. If $a$ is accidentally small, the order parameters (3.28) and (3.29) look very similar and would display similar superconducting properties. 


\section{EPJ Web of Conferences}

\section{Sensitivity to the disorder}

In pure metals, the momentum $\boldsymbol{k}$ of the electrons is well defined. However, in real metals electrons are scattered over impurities and $\boldsymbol{k}$ is not a good quantum label for electronic states. In the diffusive limit, the electronic properties result from all the contributions on the Fermi surface. The corresponding momentum averaging is harmless for superconductivity if the order parameter is isotropic, that is for conventional superconductivity with a constant order parameter over the Fermi surface. For instance, the critical temperature does not depend on the concentration of non-magnetic impurities.

Instead, the average over the Fermi surface of the order parameter in unconventional superconductors vanishes:

$$
\int \frac{d \Omega_{\hat{k}}}{4 \pi} g(\boldsymbol{k})=0 \quad \text { or } \quad \int \frac{d \Omega_{\hat{k}}}{4 \pi} d(k)=0 .
$$

This suggests that the isotropization of the electronic properties in the presence of a non-magnetic disorder leads to the suppression of the critical temperature. In practice, this occurs when the mean-free path, $\ell \sim v_{F} \tau$, due to the collisions over the impurities ( $\tau$ is the scattering time) is larger than the Copper pair size, $\xi \sim \hbar v_{F} / \Delta$.

\subsection{Symmetry lines and points of nodes}

\subsubsection{Nodes in the order parameter}

As we just illustrated, the choice of the basis functions in an IR is somewhat arbitrary in the absence of a microscopic model. However, some properties of the order parameter are independent on it. For instance, let us consider the (unspecified) order parameter $g(\hat{\boldsymbol{k}})$ associated with the singlet state corresponding to $\Gamma=B_{1 g}$ in a tetragonal crystal. Applying the symmetry operation $U_{1}$ we first obtain $g\left(\hat{k}_{x}, \hat{k}_{y}, \hat{k}_{z}\right)=$ $-g\left(\hat{k}_{y}, \hat{k}_{x},-\hat{k}_{z}\right)$. Then, applying the inversion $I$, we get $-g\left(\hat{k}_{y}, \hat{k}_{x},-\hat{k}_{z}\right)=-g\left(-\hat{k}_{y},-\hat{k}_{x}, \hat{k}_{z}\right)$. Finally, applying the rotation $C_{2}$ we get $-g\left(-\hat{k}_{y},-\hat{k}_{x}, \hat{k}_{z}\right)=-g\left(\hat{k}_{y}, \hat{k}_{x}, \hat{k}_{z}\right)$. Inserting $\hat{k}_{x}=\hat{k}_{y}$ in the resulting identity $g\left(\hat{k}_{x}, \hat{k}_{y}, \hat{k}_{z}\right)=-g\left(\hat{k}_{y}, \hat{k}_{x}, \hat{k}_{z}\right)$, we find

$$
g\left(\hat{k}_{x}, \hat{k}_{x}, \hat{k}_{z}\right)=0 .
$$

That is, the order parameter vanishes on the Fermi surface along the line $\hat{k}_{x}=\hat{k}_{y}$. The specific choice (3.28) indeed accounts for this result.

Such a discussion can be extended to any IR. As a result, the order parameter in a specific IR can be characterized by lines and/or points nodes that are imposed by the symmetries. Note however that "accidental" nodes may also appear. This is the case for the order parameter (3.29) in the representation $A_{1 g}$, at $|a|<|b|$. Note also that isolated points of nodes at the poles of the Fermi sphere are found in the A-phase of superfluid ${ }^{3} \mathrm{He}$, and that unconventional superfluids or superconductors do not necessarily contain nodes (cf. the B-phase in ${ }^{3} \mathrm{He}$ ).

The presence of nodes has important consequences on the thermodynamic and transport properties. To discuss them, we need to introduce the microscopic BCS theory generalized to unconventional superconductors. For this, we will make use of the formalism of the second quantization.

\subsubsection{Elementary excitations}

Interacting electrons can be described with the following Hamiltonian written in the grand canonical ensemble:

$$
\mathcal{H}=\sum_{\boldsymbol{k}, \sigma=\uparrow, \downarrow} \xi_{\boldsymbol{k}} c_{\boldsymbol{k} \sigma}^{\dagger} c_{\boldsymbol{k} \sigma}+\frac{1}{2} \sum_{\sigma_{1} \sigma_{2} \sigma_{3} \sigma_{4}} \sum_{\boldsymbol{k}, \boldsymbol{k}^{\prime}, \boldsymbol{q}} V_{\sigma_{1} \sigma_{2} \sigma_{3} \sigma_{4}}\left(\boldsymbol{k}, \boldsymbol{k}^{\prime}\right) c_{\boldsymbol{k} \sigma_{1}}^{\dagger} c_{-\boldsymbol{k}+\boldsymbol{q} \sigma_{2}}^{\dagger} c_{-\boldsymbol{k}^{\prime}-\boldsymbol{q} \sigma_{3}} c_{\boldsymbol{k}^{\prime} \sigma_{4}} .
$$




\section{Contribution of Symmetries in Condensed Matter}

Here, $c_{\boldsymbol{k} \sigma}$ and $c_{\boldsymbol{k} \sigma}^{\dagger}$ are the fermionic annihilation and creation operators of electrons with momentum $\boldsymbol{k}$ and spin $\sigma$, which have a kinetic energy $\xi_{k}$ measured from the Fermi level. They obey the fermionic anticommutation rules:

$$
\begin{aligned}
& \left\{c_{\boldsymbol{k} \sigma}, c_{\boldsymbol{k}^{\prime} \sigma^{\prime}}^{\dagger}\right\}=\delta_{\sigma \sigma^{\prime}} \delta_{\boldsymbol{k} \boldsymbol{k}^{\prime}}, \\
& \left\{c_{\boldsymbol{k} \sigma}, c_{\boldsymbol{k}^{\prime} \sigma^{\prime}}\right\}=0, \\
& \left\{c_{\boldsymbol{k} \sigma}^{\dagger}, c_{\boldsymbol{k}^{\prime} \sigma^{\prime}}^{\dagger}\right\}=0 .
\end{aligned}
$$

The second term in Eq. (3.32) describes a two-body interaction depending on the relative distance between the electrons. On the whole, Eq. (3.32) generalizes the Hamiltonian entering the Schrödinger equation (3.1) to arbitrary crystal structures. The second quantized form allows describing without much effort many-body wavefunctions for fermionic particles, while Eq. (3.1) was limited to a two-body wavefunction.

To simplify the discussion, we only consider the singlet case:

$$
V_{\sigma_{1} \sigma_{2} \sigma_{3} \sigma_{4}}\left(\boldsymbol{k}, \boldsymbol{k}^{\prime}\right)=\frac{1}{2}\left[i \sigma_{y}\right]_{\sigma_{1} \sigma_{2}}\left[i \sigma_{y}\right]_{\sigma_{3} \sigma_{4}}^{\dagger} V\left(\boldsymbol{k}, \boldsymbol{k}^{\prime}\right)
$$

Equation (3.32) can thus be written

$$
\mathcal{H}=\sum_{\boldsymbol{k}, \sigma=\uparrow, \downarrow} \xi_{\boldsymbol{k}} c_{\boldsymbol{k} \sigma}^{\dagger} c_{\boldsymbol{k} \sigma}+\sum_{\boldsymbol{k}, \boldsymbol{k}^{\prime}} V\left(\boldsymbol{k}, \boldsymbol{k}^{\prime}\right) c_{\boldsymbol{k} \uparrow}^{\dagger} c_{-\boldsymbol{k}+\boldsymbol{q} \downarrow}^{\dagger} c_{-\boldsymbol{k}^{\prime}-\boldsymbol{q} \downarrow} c_{\boldsymbol{k}^{\prime} \uparrow} .
$$

In order to diagonalize the Hamiltonian (3.35), a mean field approximation taking into account the pairing of electrons with opposite momenta can be employed by making use of the identities

$$
\begin{aligned}
c_{\boldsymbol{k} \uparrow}^{\dagger} c_{-\boldsymbol{k} \downarrow}^{\dagger} & =\left\langle c_{\boldsymbol{k} \uparrow}^{\dagger} c_{-\boldsymbol{k} \downarrow}^{\dagger}\right\rangle+\left(c_{\boldsymbol{k} \uparrow}^{\dagger} c_{-\boldsymbol{k} \downarrow}^{\dagger}-\left\langle c_{\boldsymbol{k} \uparrow}^{\dagger} c_{-\boldsymbol{k} \downarrow}^{\dagger}\right\rangle\right), \\
c_{-\boldsymbol{k}^{\prime} \downarrow} c_{\boldsymbol{k}^{\prime} \uparrow} & =\left\langle c_{-\boldsymbol{k}^{\prime} \downarrow} c_{\boldsymbol{k}^{\prime} \uparrow}\right\rangle+\left(c_{-\boldsymbol{k}^{\prime} \downarrow} c_{\boldsymbol{k}^{\prime} \uparrow}-\left\langle c_{-\boldsymbol{k}^{\prime} \downarrow} c_{\boldsymbol{k}^{\prime} \uparrow}\right\rangle\right) .
\end{aligned}
$$

Here, the brackets denote a quantum statistical averaging on the eigenstates of the Hamiltonian (3.35). The average $\left\langle c_{\boldsymbol{k} \uparrow}^{\dagger} c_{-\boldsymbol{k} \downarrow}^{\dagger}\right\rangle$, with the combination of two creation operators, reflects that a macroscopic number of Cooper pairs have been formed. As the Hamiltonian (3.32) is particle-conserving, such an average would apparently vanish in the canonical ensemble, at fixed particle number. The Bose-like condensation of Cooper pairs is more conveniently dealt with in the grand canonical ensemble, at fixed chemical potential, without the need of specifying the number of electrons in the states over which the average is performed.

Inserting Eqs. (3.36) into (3.35), while assuming that the terms between parentheses are small, and retaining the leading-order terms, we obtain a quadratic Hamiltonian in the creation and annihilation operators,

$$
\begin{aligned}
\mathcal{H} & =\sum_{k, \sigma=\uparrow, \downarrow} \xi_{k} c_{k \sigma}^{\dagger} c_{k \sigma}+\sum_{k}\left[\Delta_{k} c_{k \uparrow}^{\dagger} c_{-k \downarrow}^{\dagger}+\Delta_{k}^{*} c_{-k \downarrow} c_{k \uparrow}-\Delta_{k}\left\langle c_{k \uparrow}^{\dagger} c_{-k \downarrow}^{\dagger}\right\rangle\right] \\
& =\sum_{k}\left(c_{k \uparrow}^{\dagger} c_{-k \downarrow}\right)\left(\begin{array}{cc}
\xi_{k} & \Delta_{k} \\
\Delta_{k}^{*} & -\xi_{k}
\end{array}\right)\left(\begin{array}{c}
c_{k \uparrow} \\
c_{-k \downarrow}^{\dagger}
\end{array}\right)-\sum_{k} \Delta_{k}\left\langle c_{k \uparrow}^{\dagger} c_{-k \downarrow}^{\dagger}\right\rangle+\sum_{k} \xi_{k} .
\end{aligned}
$$

where the variational field

$$
\Delta_{k}=\sum_{\boldsymbol{k}^{\prime}} V\left(\boldsymbol{k}, \boldsymbol{k}^{\prime}\right)\left\langle c_{-\boldsymbol{k}^{\prime} \downarrow} c_{\boldsymbol{k}^{\prime} \uparrow}\right\rangle
$$


has been introduced. The Hamiltonian (3.37) does not conserve the particle number, but it can be easily diagonalized into

$$
\mathcal{H}=\sum_{k, \sigma} E_{k} \gamma_{k \sigma}^{\dagger} \gamma_{k \sigma}+\sum_{k}\left[-\Delta_{k}\left\langle c_{k \uparrow}^{\dagger} c_{-k \downarrow}^{\dagger}\right\rangle+\xi_{k}-E_{k}\right],
$$

after the unitary transformation (called a Bogoliubov transformation)

$$
c_{k \sigma}=u_{k} \gamma_{k \sigma}-\sigma v_{k} \gamma_{-k-\sigma}^{\dagger} .
$$

Here, $\gamma_{k \sigma}$ and $\gamma_{k \sigma}^{\dagger}$ are the annihilation and creation operators for the quasiparticles in superconducting state, with excitation energy

$$
E_{k}=\sqrt{\xi_{k}^{2}+\left|\Delta_{k}\right|^{2}}
$$

$u_{k}=\left[\frac{1}{2}\left(1+\xi_{k} / E_{k}\right)\right]^{1 / 2}$ and $v_{k}=e^{i \phi_{k}}\left[\frac{1}{2}\left(1-\xi_{k} / E_{k}\right)\right]^{1 / 2}$ are the coherence factors, and $\phi_{k}=\arg \Delta_{k}{ }^{8}$ The occupation of the quasiparticle states is given by

$$
\left\langle\gamma_{k \sigma}^{\dagger} \gamma_{k \sigma}\right\rangle=f\left(E_{k}\right) \equiv f_{k},
$$

where $f(E)=1 /\left(1+e^{E / k_{B} T}\right)$ is the Fermi distribution function at temperature $T$. The self-consistency equation (3.38) for the order parameter reads

$$
\Delta_{k}=-\sum_{\boldsymbol{k}^{\prime}} V\left(\boldsymbol{k}, \boldsymbol{k}^{\prime}\right) \frac{\Delta_{\boldsymbol{k}^{\prime}}}{E_{\boldsymbol{k}^{\prime}}}\left(1-2 f_{\boldsymbol{k}^{\prime}}\right)
$$

Like in Eqs. (3.5)-(3.6) for the isotropic case, the attractive potential giving the highest critical temperature in the IR $\Gamma$ can be decomposed into

$$
V\left(\boldsymbol{k}, \boldsymbol{k}^{\prime}\right)=V_{\Gamma}\left(k, k^{\prime}\right) \sum_{i=1}^{d_{\Gamma}} \psi_{i}^{\Gamma}(\hat{\boldsymbol{k}})^{*} \psi_{i}^{\Gamma}\left(\hat{\boldsymbol{k}}^{\prime}\right),
$$

with $V_{\Gamma}\left(k, k^{\prime}\right)=-\left|V_{\Gamma}\right|$ if $\left|\xi_{k}\right|,\left|\xi_{k^{\prime}}\right|<\hbar \Omega_{\Gamma}$, and $V_{\Gamma}\left(k, k^{\prime}\right)=0$ otherwise. We also decompose the order parameter into $\Delta_{k}=\Delta(T) g(\hat{\boldsymbol{k}})$, where $g(\hat{\boldsymbol{k}})=\sum_{i=1}^{d_{\Gamma}} \eta_{i} \psi_{i}^{\Gamma}(\hat{\boldsymbol{k}})$ (cf. Eq. (3.19)) is now chosen to be normalized:

$$
\int \frac{d \Omega_{\hat{k}}}{4 \pi}|g(\hat{k})|^{2}=1
$$

Equation (3.43) then defines a non-linear set of coupled equations for the components $\eta_{i}$ of the order parameter and its amplitude $\Delta(T)$ at temperature $T$ :

$$
\eta_{i}=\left|V_{\Gamma}\right| \sum_{\boldsymbol{k}} \sum_{j} \frac{\eta_{j} \psi_{i}^{\Gamma}(\hat{\boldsymbol{k}})^{*} \psi_{j}^{\Gamma}(\hat{\boldsymbol{k}})}{\sqrt{\xi_{\boldsymbol{k}}^{2}+\Delta(T)^{2}|g(\hat{\boldsymbol{k}})|^{2}}} \tanh \left(\frac{\sqrt{\xi_{\boldsymbol{k}}^{2}+\Delta(T)^{2}|g(\hat{\boldsymbol{k}})|^{2}}}{2 k_{B} T}\right) .
$$

This complicated equation simplifies at $T \rightarrow T_{c}(\Gamma)$, close to the superconducting transition, when $\Delta(T) \rightarrow 0$. It allows finding the critical temperature with the equation

$$
1=\left|V_{\Gamma}\right| \sum_{k} \frac{\tanh \left[\xi_{k} / 2 k_{B} T_{c}(\Gamma)\right]}{\xi_{k}} .
$$

\footnotetext{
8 In the normal state with $\Delta_{k}=0$, the Hamiltonian for bare electrons is transformed into a Hamiltonian for quasi-particles with positive excitation energy, $E_{k}=\left|\xi_{k}\right|$ under such a transformation. It describes quasiparticles of the particle type $\left(\xi_{k}>0\right)$ and of the hole type $\left(\xi_{k}<0\right)$.
} 


\section{Contribution of Symmetries in Condensed Matter}

The solution is

$$
k_{B} T_{c}(\Gamma) \approx 1.14 \hbar \Omega_{\Gamma} \exp \left(-\frac{1}{v_{0}\left|V_{\Gamma}\right|}\right),
$$

which looks similar to the result expected in Sec. 3.1. Calculating $\Delta(T)$ at arbitrary temperature is a much harder task. Nevertheless, the end result at $T=0$ scales as $\Delta(0) \sim k_{B} T_{c}(\Gamma)$.

The above theory can be extended to triplet superconductors as well. An excitation spectrum (3.41) with $\Delta_{\boldsymbol{k}}=\Delta(T) \boldsymbol{d}(\hat{\boldsymbol{k}}) \cdot \boldsymbol{\sigma}$ is found, provided $\boldsymbol{d}(\hat{\boldsymbol{k}}) \times \boldsymbol{d}(\hat{\boldsymbol{k}})^{*}=0$, while the general case is more complicated and won't be addressed in these notes [5].

Density of states. The energy spectrum (3.41) gives a density of states

$$
v(E)=\sum_{k} \delta\left(E-E_{k}\right)=2 v_{0} \int \frac{d \Omega_{\hat{k}}}{4 \pi} \frac{E}{\sqrt{E^{2}-\left|\Delta_{k}\right|^{2}}} \theta\left(E-\left|\Delta_{k}\right|\right),
$$

where $\theta(x)=1$ if $x>0$, and $\theta(x)=0$ if $x<0$. For superconducting states with a constant gap $\left|\Delta_{k}\right|=\Delta$ (in particular, for conventional superconductors), the density of states vanishes at energies $E<\Delta$. Instead, the order parameters that vanish for specific directions allow quasiparticle states to exist at energies arbitrarily close to the Fermi level. For instance, in the high-temperature superconductors with d-wave symmetry, the main contribution to the density of states at low energies $E \ll \Delta$ comes from the region over the Fermi surface where the order parameter (3.28) vanishes,

$$
\nu(E) \propto \nu_{0} \int_{0}^{E / \Delta} d \varphi \frac{E}{\sqrt{E^{2}-\Delta^{2} \varphi^{2}}} \sim \nu_{0} \frac{E}{\Delta} .
$$

Such a power law is actually characteristic of order parameters that have lines of nodes. The density of states at low energy for an order parameter having nodes at isolated points is $v(E) \sim v_{0}(E / \Delta)^{2}$.

The density of states can be accessed experimentally by measuring the tunneling current between the superconductor and a normal metal or by Angle-Resolved Photoemission Electron Spectroscopy (ARPES). Experiments in high- $T_{c}$ superconductors have confirmed the prediction (3.50), thus giving credit to an order parameter with d-wave symmetry.

Specific heat. We now illustrate that low-temperature properties in superconducting state are also strongly influenced by the presence or absence of nodes in the gap.

For instance, let us consider the specific heat. Using the definition $C=T(\partial S / \partial T)$, where

$$
S=-k_{B} \sum_{k, \sigma}\left[f_{k} \ln f_{k}+\left(1-f_{k}\right) \ln \left(1-f_{k}\right)\right]
$$

is the entropy, we obtain

$$
C=2 \sum_{k} \frac{E_{k}^{2}}{\left(k_{B} T\right)^{2}} \frac{e^{-E_{k} / k_{B} T}}{\left(1+e^{-E_{k} / k_{B} T}\right)^{2}} .
$$

Here, the factor 2 comes from spin degeneracy. In normal state, we evaluate Eq. (3.52) with the spectrum $E_{k}=\left|\xi_{k}\right|$ and we find that it varies linearly with the temperature, $C_{n}=\left(\pi^{2} / 3\right) v_{0} k_{B} T$. In a gapped spectrum, with gap $\Delta$, only thermally excited quasiparticles with small energy, $E_{k} \simeq \Delta+\xi_{k}^{2} / 2 \Delta$, contribute to Eq. (3.52) at low temperature, with the result

$$
C_{s} / C_{n} \sim\left(\Delta / k_{B} T\right)^{5 / 2} e^{-\Delta / k_{B} T} .
$$

Therefore, the specific heat is exponentially suppressed at low temperatures. Instead, if the order parameter contains nodes, the specific heat will obey a power-law with the temperature. To be specific, let us again consider the d-wave state (3.28) that has lines of nodes. The main contribution to Eq. (3.52), 
or equivalently

$$
C_{s}=2 \int_{0}^{\infty} d E v(E) \frac{E^{2}}{4 k_{B}^{2} T^{2} \cosh ^{2}\left(E / 2 k_{B} T\right)},
$$

comes from the regions of the Fermi surface where the order parameter vanishes. Using Eq. (3.50), we find

$$
C_{s} / C_{n} \sim T / T_{c}
$$

By contrast, isolated nodes would result in a different power law, $C_{s} / C_{n} \sim\left(T / T_{c}\right)^{2}$.

Such power-laws have been predicted and observed in the temperature dependence of several thermodynamic and transport properties in high- $T_{c}$ superconductors.

\subsection{Phenomenological theory of unconventional superconductors}

Many features discussed within the microscopic model depend on the specific choice of the basis functions. Moreover, the BCS theory can be easily solved only under several crude approximations.

By contrast, the phenomenological Ginzburg-Landau theory is quite versatile. ${ }^{9}$ In particular, it applies even if the specific microscopic model for superconductivity is not known. In this section, we propose a generalized GL theory that applies to unconventional superconductors. We discuss its implications and point out the differences with the conventional GL theory of Sec. 2.

The free energy per unit volume for a multidimensional order parameter $\boldsymbol{\eta}=\left\{\eta_{i}\right\}$ with dimension $d_{\Gamma}$ is

$$
F[\boldsymbol{\eta}]=F_{n}+\alpha \sum_{i}\left|\eta_{i}\right|^{2}+\frac{1}{2} \sum_{i_{1} i_{2} i_{3} i_{4}} \beta_{i_{1} i_{2} i_{3} i_{4}} \eta_{i_{1}}^{*} \eta_{i_{2}}^{*} \eta_{i_{3}} \eta_{i_{4}}+\sum_{i_{1} i_{2} i_{3} i_{4}} K_{i_{1} i_{2} i_{3} i_{4}}\left(D_{i_{1}} \eta_{i_{2}}\right)^{*}\left(D_{i_{3}} \eta_{i_{4}}\right)+\frac{\boldsymbol{h}^{2}}{8 \pi}
$$

where we used the short notation $\boldsymbol{D}=-i \boldsymbol{\nabla}-(2 e / \hbar c) \boldsymbol{A}$. The tensors $\beta_{i_{1} i_{2} i_{3} i_{4}}$ and $K_{i_{1} i_{2} i_{3} i_{4}}$ are constrained by the symmetry of the superconducting crystal. For one-dimensional order parameters, the quadratic and quartic terms in Eq. (3.56) coincide with those in (2.7). The only difference comes from the gradient term that is more general here. For multidimensional order parameters, the free energy (3.56) can describe several superconducting states, all with the same critical temperature.

\subsubsection{Multicomponent GL theory}

We consider a tetragonal crystal with singlet Cooper pairs. Focussing on the two-dimensional representation $\Gamma=E_{g}$ (see Table 1), we decompose the order parameter as

$$
g(\hat{\boldsymbol{k}})=\eta_{x} \hat{k}_{x} \hat{k}_{z}+\eta_{y} \hat{k}_{y} \hat{k}_{z} .
$$

The most general free energy at zero magnetic field reads:

$$
F\left[\eta_{x}, \eta_{y}\right]=F_{n}+\alpha\left(\left|\eta_{x}\right|^{2}+\left|\eta_{y}\right|^{2}\right)+\beta_{1}\left(\left|\eta_{x}\right|^{2}+\left|\eta_{y}\right|^{2}\right)^{2}+\beta_{2}\left|\eta_{x}^{2}+\eta_{y}^{2}\right|^{2}+\beta_{3}\left(\left|\eta_{x}\right|^{4}+\left|\eta_{y}\right|^{4}\right) .
$$

Stable solutions are found by minimizing Eq. (3.58). Three different phases may compete:

- The A-phase corresponds to an order parameter $\left(\eta_{x}, \eta_{y}\right) \propto(1,0)$ or $(0,1)$, that is $g(\hat{\boldsymbol{k}})=\eta \hat{k}_{x} \hat{k}_{z}$ or $\eta \hat{k}_{y} \hat{k}_{z}$. Thus it has two lines of nodes at $k_{x}=0, k_{z}=0$ or $k_{y}=0, k_{z}=0$. The free energy in these states is $F=F_{n}-\alpha^{2} / 2\left(\beta_{1}+\beta_{2}+\beta_{3}\right)$;

- The B-phase corresponds to an order parameter $\left(\eta_{x}, \eta_{y}\right) \propto(1,1)$ or $(1,-1)$, that is $g(\hat{\boldsymbol{k}})=\eta\left(\hat{k}_{x} \pm\right.$ $\left.\hat{k}_{y}\right) \hat{k}_{z}$. It has also two lines of nodes at $k_{x}=-k_{y}, k_{z}=0$ or $k_{x}=k_{y}, k_{z}=0$. The free energy in these states is $F=F_{n}-\alpha^{2} / 2\left(\beta_{1}+\beta_{2}+\beta_{3} / 2\right)$;

\footnotetext{
${ }^{9}$ It is eventually derived with the microscopic BCS theory.
} 


\section{Contribution of Symmetries in Condensed Matter}

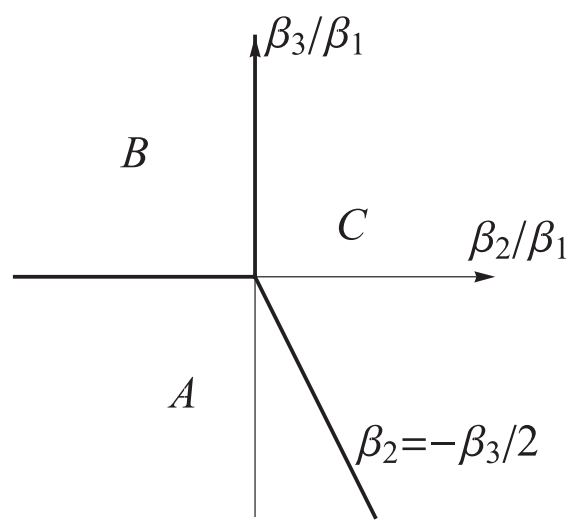

Figure 4. Phase diagram of two-component superconducting states in a tetragonal crystal.

- The C-phase corresponds to $\left(\eta_{x}, \eta_{y}\right) \propto(1, \pm i)$, that is $g(\hat{\boldsymbol{k}})=\eta\left(\hat{k}_{x} \pm i \hat{k}_{y}\right) \hat{k}_{z}$. It has one line of nodes at $k_{z}=0$ and two points of nodes at the poles of the Fermi sphere. Moreover, time-reversal symmetry is broken in these states as they bear an orbital magnetism. The free energy is $F=$ $F_{n}-\alpha^{2} / 2\left(\beta_{1}+\beta_{3} / 2\right)$.

While each of these phases has the same critical temperature, their free energies are different. Which of them is actually more stable depends on the relative values of the GL parameters $\beta_{1}, \beta_{2}$, and $\beta_{3}$, see Fig. 4. Moreover, two possible solutions have been found in each phase. This means that domains can form and they would be separated by domain walls.

\subsubsection{Anisotropy of the upper critical field}

In Sec. 2.3, we determined the upper critical field $H_{c 2}$ in type-II superconductors with the conventional GL theory. We generalize this calculation to the free energy (3.56) and we discuss the anisotropy of $H_{c 2}$ in two different cases.

Uniaxial crystal with one-component superconducting state. The free energy reads

$$
F[\eta]=F_{n}+\alpha|\eta|^{2}+\frac{\beta}{2}|\eta|^{4}+K_{1}\left|\boldsymbol{D}_{\perp} \eta\right|^{2}+K_{4}\left|D_{z} \eta\right|^{2}+\frac{\boldsymbol{h}^{2}}{8 \pi} .
$$

We assume that the magnetic field is applied in the $(y, z)$-plane, $\boldsymbol{H}=H(0, \sin \theta, \cos \theta)$. A convenient gauge is $\boldsymbol{A}=H x(0,-\cos \theta, \sin \theta)$, so that the linearized self-consistency equation for the order parameter at the second order phase transition reads

$$
-K_{1} \frac{\partial^{2} \eta}{\partial x^{2}}+\left[K_{1} \cos ^{2} \theta+K_{4} \sin ^{2} \theta\right]\left(\frac{2 \pi H}{\Phi_{0}}\right)^{2} x^{2} \eta=-\alpha \eta .
$$

This equation allows determining the upper critical field

$$
H_{c 2}(T)=\frac{\Phi_{0} \alpha_{0}}{2 \pi T_{c} \sqrt{K_{1}\left[K_{1} \cos ^{2} \theta+K_{4} \sin ^{2} \theta\right]}}\left(T_{c}-T\right) .
$$

In contrast, there is no anisotropy in the basal plane $(x, y)$. The ratio between the upper critical fields applied parallel or perpendicular to the basal plane is independent of the temperature, $H_{c 2}^{\|} / H_{c 2}^{\perp}=$ $\left(K_{1} / K_{4}\right)^{1 / 2}$. 
Tetragonal crystal with two-component superconducting state. For the representation $E_{g}$, the quadratic terms in the free energy read

$$
\begin{aligned}
F[\eta]= & F_{n}+\alpha\left(\left|\eta_{x}\right|^{2}+\left|\eta_{y}\right|^{2}\right)+\sum_{i, j=x, y}\left[K_{1}\left(D_{i} \eta_{j}\right)^{*}\left(D_{i} \eta_{j}\right)+K_{2}\left(D_{i} \eta_{i}\right)^{*}\left(D_{j} \eta_{j}\right)\right. \\
& \left.+K_{3}\left(D_{i} \eta_{j}\right)^{*}\left(D_{j} \eta_{i}\right)\right]+\sum_{i=x, y}\left[K_{4}\left(D_{z} \eta_{i}\right)^{*}\left(D_{z} \eta_{i}\right)+K_{5}\left(D_{i} \eta_{i}\right)^{*}\left(D_{i} \eta_{i}\right)\right]
\end{aligned}
$$

To simplify the discussion, let us first assume that $K_{2}=K_{3}=0$. We also consider a magnetic field applied in the $(x, y)$-plane, $\boldsymbol{H}=H(\cos \varphi, \sin \varphi, 0)$. A convenient gauge is $\boldsymbol{A}=H z(-\sin \varphi, \cos \varphi, 0)$, so that the linearized self-consistency equations for the order parameter at the second order phase transition read

$$
\left\{\begin{array}{l}
-K_{4}\left(\partial^{2} \eta_{x} / \partial z^{2}\right)+\left[\left(K_{1}+K_{5}\right) \sin ^{2} \varphi+K_{1} \cos ^{2} \varphi\right]\left(2 \pi H / \Phi_{0}\right)^{2} z^{2} \eta_{x}=-\alpha \eta_{x} \\
-K_{4}\left(\partial^{2} \eta_{y} / \partial z^{2}\right)+\left[\left(K_{1}+K_{5}\right) \cos ^{2} \varphi+K_{1} \sin ^{2} \varphi\right]\left(2 \pi H / \Phi_{0}\right)^{2} z^{2} \eta_{y}=-\alpha \eta_{y} .
\end{array}\right.
$$

Thus, $\eta_{x}$ and $\eta_{y}$ are linearly independent from each other and they obey different equations. We thus find that an A-phase is realized at finite magnetic field, with upper critical field

$$
H_{c 2}^{(x)}(T)=\frac{\Phi_{0} \alpha_{0}}{2 \pi T_{c} \sqrt{K_{4}\left[K_{1}+K_{5} \sin ^{2} \varphi\right]}}\left(T_{c}-T\right)
$$

for $\left(\eta_{x}, \eta_{y}\right) \propto(1,0)$, or

$$
H_{c 2}^{(y)}(T)=\frac{\Phi_{0} \alpha_{0}}{2 \pi T_{c} \sqrt{K_{4}\left[K_{1}+K_{5} \cos ^{2} \varphi\right]}}\left(T_{c}-T\right)
$$

for $\left(\eta_{x}, \eta_{y}\right) \propto(0,1)$. The actual critical field is the highest of them. As a result, $H_{c 2}$ shows a strong anisotropy in the basal plane, with a kink at angles $\varphi=\pi / 4+k \pi$, when $H_{c 2}^{(x)}$ and $H_{c 2}^{(y)}$ are degenerate.

We also notice that the A-phase may be unstable at zero magnetic field, depending on the coefficients in front of the quartic terms in the free energy (3.58), cf. Sec. 3.5.1. We would then expect a complicated $(T, H)$-phase diagram in this case.

A formula for the upper critical field at finite coefficients $K_{2}$ and $K_{3}$, thus generalizing Eqs. (3.64), can also be obtained:

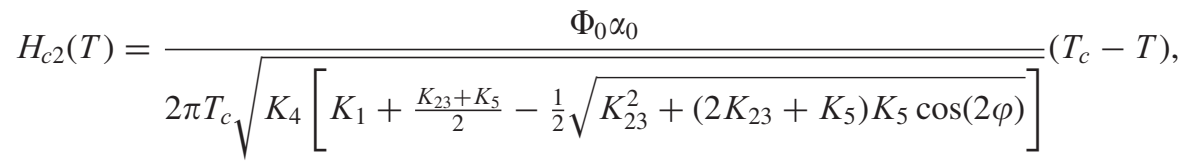

where $K_{23}=K_{2}+K_{3}$. The anisotropy of $H_{c 2}$ in the basal plane is illustrated in Fig. 5, it is entirely due to the coefficient proportional to $K_{5}$ in the free energy (3.62).

\subsubsection{Josephson effect}

As we discussed in Sec. 2.4, the GL theory can be extended to describe Josephson junctions by adding a boundary energy term to the free energy. In unconventional superconductors, the amplitude of the critical current depends on the orientation of the plane of the junction with respect to the crystal axes in the leads. Such an orientation can be parametrized with the unit vector $\hat{\boldsymbol{n}}$ perpendicular to the plane of the junction. In a junction between two even, one-dimensional superconductors with order parameters $\eta_{1}$ and $\eta_{2}$, the boundary energy term (2.29) should be substituted with

$$
F_{12}=\gamma \int d \boldsymbol{x} f_{1}(\hat{\boldsymbol{n}}) f_{2}(\hat{\boldsymbol{n}})\left[\eta_{1}^{*} \eta_{2}+\eta_{2}^{*} \eta_{1}\right] \delta(x),
$$




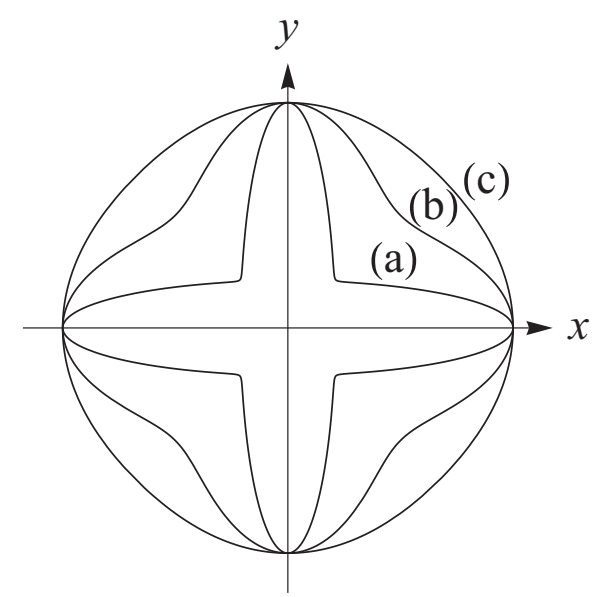

Figure 5. Anisotropy of the upper critical field in the basal plane for the two-component superconducting state in a tetragonal crystal. The curves correspond to $H_{c 2}(\varphi) / H_{c 2}(\varphi=0)$ at $K_{1}=K_{23}$ and $K_{5} / K_{23}=10$ (a), 1 (b), and 0.1 (c).

where $f_{1}(\hat{\boldsymbol{n}})$ and $f_{2}(\hat{\boldsymbol{n}})$ are scalar functions of $\hat{\boldsymbol{n}}$ and each of the products $f_{1}(\hat{\boldsymbol{n}}) \eta_{1}$ and $f_{2}(\hat{\boldsymbol{n}}) \eta_{2}$ should be invariant under all the symmetry operations in the corresponding superconducting class. Thus, $f_{1}(\hat{\boldsymbol{n}})$ and $f_{2}(\hat{\boldsymbol{n}})$ simply have the same symmetry as $\eta_{1}$ and $\eta_{2}$. For a conventional, isotropic superconductor, we thus get $f(\hat{\boldsymbol{n}})=1$, as expected; for the d-wave superconductors, we can choose $f(\hat{\boldsymbol{n}})=\hat{n}_{x}^{2}-\hat{n}_{y}^{2}$.

The Josephson current can be derived as in Sec. 2.4. In particular, we find that the critical current between a s-wave and d-wave superconductors is

$$
I_{c}=(4 e / \hbar) \gamma S\left|\eta_{1}\right|\left|\eta_{2}\right|\left(\hat{n}_{x}^{2}-\hat{n}_{y}^{2}\right)
$$

It vanishes when the plane of the junction makes an angle $\pi / 4$ with respect to the crystal axes. Such a property was used to build a convincing test of the d-wave symmetry of the order parameter in high- $T_{c}$ compounds.

\section{CONCLUSION AND PERSPECTIVES}

Superconductivity is still an active field of research: The quest for new materials with higher critical temperatures, as well as for new microscopic theories that would allow understanding how to get them, still continues. In a different field, the progress of microtechnology now allows building complex circuits with conventional superconducting elements on the nanometer scale. This research allows addressing questions related with the crossover from the quantum microscopic to classical macroscopic world.

The progress in fabrication and the discovery of new materials in recent years called for important developments in the field of unconventional superconductivity. Let us list a few of them:

- Noncentrosymmetric superconductors, which do not possess an inversion center, have been discovered. There, Eq. (3.18) cannot be used anymore, and the superconducting order parameter exhibits singlet-triplet mixing. This could result in a spontaneously modulated superconducting state [10].

- The temperature and pressure phase diagram of a ferromagnetic superconductor (FS) revealed a tetracritical point where the normal, ferromagnetic (F), superconducting (S) and FS states coexist. The symmetry analysis has been extended to discuss the $\mathrm{F} \rightarrow \mathrm{FS}$ and $\mathrm{S} \rightarrow \mathrm{FS}$ transitions [11].

- Multiband superconductivity with bands of different symmetries can give rise to "unconventional" superconducting properties, provided that the conventional order parameters in each band are 
Josephson-coupled in such a way that they have opposite signs ( $\pi$-coupling). Such a scenario was discussed in relation with the recently discovered family of iron-pnictide superconductors [12].

- The injection of singlet Cooper pairs from a conventional superconductor into triplet pairs of electrons with parallel spins, which propagate coherently over long distances, in a ferromagnetic metal with a non colinear domain structure has been demonstrated [13]. ${ }^{10}$

- There is an active search to observe topological superconductors that admit zero-energy Majorana states (fermions that are their own antiparticle) localized at their interfaces while their bulk spectrum is gapped. Such systems could be realized by contacting conventional superconductors with the recently discovered topological insulators that - unlike ordinary insulators - admit robust conducting states at their edges [14].

These examples are indications that many discoveries in the field of superconductivity are yet to come. In parallel with the ongoing search for a microscopic theory of superconductivity in these systems, the symmetry analysis shall remain an important guide in the understanding of their properties.

I thank V. Mineev and P. Rodière for a critical reading of the notes and useful suggestions.

\section{References}

[1] Superconductivity of metals and alloys, P. G. De Gennes (Cambridge, MA : Perseus, 1999).

[2] Introduction to superconductivity, M. Tinkham (McGraw-Hill: New York, 1996).

[3] Fundamentals of the theory of metals, A. A. Abrikosov (North-Holland, Elsevier Science Publishers, B.V., Amsterdam, 1988).

[4] Phenomenological theory of unconventional superconductivity, M. Sigrist and K. Ueda, Rev. Mod. Phys. 63, 239 (1991).

[5] Introduction to unconventional superconductivity, V. P. Mineev and K. V. Samokhin (Gordon and Breach Science, Amsterdam, 1999).

[6] Generalized Ginzburg-Landau theory for nonuniform FFLO superconductors, A. I. Buzdin and H. Kachkachi, Phys. Lett. A 225, 341 (1997).

[7] Electrodynamics of continuous media, Course of theoretical physics vol. 8, E. M. Lifshitz and L. P. Pitaevskii, Pergamon Press (1984).

[8] A. Huxley, in Vortices in unconventional superconductors and superfluids, Springer Series in Solid-State Sciences, Vol. 132, R. P. Huebener, N. Schopohl, G. E. Volovik (Eds.) (2002).

[9] A theoretical description of the new phases of liquid ${ }^{3} \mathrm{He}$, A. J. Leggett, Rev. Mod. Phys. 47, 331 (1975).

[10] Introduction to superconductivity in metals without inversion center, V. P. Mineev and M. Sigrist, arXiv:0904.2962.

[11] Coexistence of triplet superconductivity and itinerant ferromagnetism, V. P. Mineev, AIP Conference Proceedings 1134, 68 (2009); arXiv:0812.2171.

[12] High-temperature superconductivity in the iron pnictides, M. R. Norman, Physics 1, 21 (2008).

[13] Spin-polarized supercurrents for spintronics, M. Eschrig, Physics Today, 64, 43 (2011).

[14] Colloquium: Topological insulators, M. Z. Hasan and C. L. Kane, Rev. Mod. Phys. 82, 3045 (2010).

\footnotetext{
${ }^{10}$ In contrast, pairs of electrons with antiparallel spins are broken by the exchange field in the ferromagnet. Thus, their quantum coherence is short ranged.
} 